\title{
Impact of Polycyclic Aromatic Hydrocarbon exposure on Cognitive Function and Neurodegeneration in Humans. A Systematic Review and Meta-Analysis
}

Jessica Humphreys ${ }^{1}$, and Maria del C. Valdés Hernández ${ }^{2}$

${ }^{1}$ College of Medicine and Veterinary Medicine, University of Edinburgh, Edinburgh, UK

${ }^{2}$ Centre for Clinical Brain Sciences, University of Edinburgh, Edinburgh, UK

Correspondence to: Dr. Maria del C. Valdés Hernández

Centre for Clinical Brain Sciences

Dementia Research Institute at The University of Edinburgh, Room FU427

Chancellor's Building

49 Little France Crescent

Edinburgh, EH16 4SB, UK

e-mail: M.Valdes-Hernan@ed.ac.uk 


\section{$\underline{\text { Abstract }}$}

This review documents an emerging body of evidence concerning the neurological effect of polycyclic aromatic hydrocarbon (PAH) exposure, with regard to cognitive function and increased risk of neurodegeneration. Two electronic databases PubMed and Web of Science were systematically searched. The $37 / 428$ studies selected included outcomes measuring cognitive function, neurobehavioral symptoms of impaired cognition, and pathologies associated with neurodegeneration from prenatal (21/37 studies), childhood (14/37 studies), and adult (8/37 studies) PAH exposure. Sufficient evidence surrounding prenatal exposure negatively impacting child intelligence, mental development, average overall development, verbal IQ, memory impairment, externalizing, internalizing, anxious, depressed behaviours, behavioural development and child attentiveness was found. Evidence concerning exposure during childhood and as an adult was scarce and highly heterogeneous, however presence of neurodegenerative biomarkers and increased concentrations of cryptic "self" antigens in serum and cerebrospinal fluid samples suggest a higher risk of neurodegenerative disease. Associations with lowered cognitive ability, and impaired attentiveness were found in children and memory disturbances, specifically auditory memory, verbal learning and general memory in adults. Although evidence is not yet conclusive and further research is needed the studies included supported the hypothesis that PAH exposure negatively impacts cognitive function and increases the risk of neurodegeneration in humans, and recommends considering the introduction of a variable "rural vs. urban" as covariate for adjusting analyses where the neurological functions affected (as result of our review) are outcome variables. Keywords: polycyclic aromatic hydrocarbons, particulate matter 2.5 (PM 2.5), cognition, neurodegeneration, neurology, air pollution, environment, rural, urban, neurocognitive development, neurodegeneration

Abbreviations

AD Alzheimer's Disease

ADHD Attention deficit hyperactivity disorder

APOE4 Apolipoprotein E4

$A \beta_{1-42}$ Amyloid beta protein fragment 1-42

$\alpha$-synuclein Alpha-synuclein

$B[a] P$ Benzo[a]pyrene

BDNF. Brain-Derived Neurotrophic Factor

CEREB IgG Cerebellar antigen

CO Carbon monoxide

CSF Cerebrospinal Fluid

ETS. Environmental Tobacco Smoke 
IFN $\gamma$ Interferon gamma

$\lg A$ ..Immunoglobulin A

$\lg G$ Immunoglobulin $\mathrm{G}$

$\lg M$ Immunoglobulin $\mathrm{M}$

IL $\beta$ Interleukin beta

IL 2 . Interleukin 2

IL 6 . Interleukin 6

IL 10 Interleukin 10

IQ. Intelligence Quotient

MBP. .Myelin Basic Protein

MBP IgA Myelin Basic Protein Immunoglobulin A

MBP IgG Myelin Basic Protein Immunoglobulin G

MCP-1 Monocyte Chemoattractant Protein-1

MOG IgG Myelin Oligodendrocyte Glycoprotein Immunoglobulin G

MOG IgM Myelin Oligodendrocyte Glycoprotein Immunoglobulin A

NHANES .National Health and Nutrition Examination Survey

Non-p-tau Non-Phosphorylated Tau

$\mathrm{NO}_{\mathrm{x}}$ Nitrogen Oxide species

$\mathrm{NO}_{2}$ Nitrogen Dioxide

OZ $\lg A$ .Occludin/ Zonulin Immunoglobulin A

$O Z \lg G$ Occludin/Zonulin Immunoglobulin G

PAH. Polycyclic Aromatic Hydrocarbons

PAH-DNA adducts....Polycyclic Aromatic Hydrocarbon- DNA adducts

PD Parkinson's Disease

PM Particulate Matter

$\mathbf{P M}_{2.5}$ ..Particulate Matter with an aerodynamic diameter $>2.5$ micrometres

$\mathrm{PM}_{10}$ Particulate Matter with an aerodynamic diameter $>10$ micrometres

p-tau. Phosphorylated Tau

S-100 IgG .Astrocytic protein Immunoglobulin $\mathrm{G}$

S-100 Ig $M$ Astrocytic protein Immunoglobulin M

TDP-43. Transactive response DNA binding protein 43

TRAP .Traffic Related Air Pollution 


\section{Introduction}

Exposure to air pollution in the environment is now recognized globally by Governments, leading research scientists, and civil society, as one of the greatest public health hazards of the $21 \mathrm{st}$ century (Cohen et al. 2017). Legislation such as "The UK National Air Quality Strategy" (Department for Environment, Food and Rural Affairs, 2011), and the European Commission's "Fourth Daughter Directive" (The European Parliament and The European Council of the European Union, 2015) have introduced standards to monitor and limit levels of air pollutants posing the greatest risk to human health. Polycyclic aromatic hydrocarbons (PAHs) are a group of pollutants included in such legislation. PAHs are atmospheric organic compounds composed of two or more benzene rings arranged in a variety of different configurations. Over 100 different PAHs have been identified to date (U.S. Department of health and human services, 1995). They are discharged from anthropogenic sources (Figure 1), involving the incomplete combustion and pyrolysis of hydrocarbons, predominantly found in: coal, oil, wood and petrol. PAHs exist in the atmosphere in a gaseous state or adsorbed to particulate matter. Over $80 \%$ of particulate-bound PAHs are associated with particulate matter of an aerodynamic diameter $\leq 2.5 \mu \mathrm{m}$ (PM2.5) (Hassanvand et al. 2015).

Research surrounding PAH exposure and acute short-term health effects in humans has thus far focused on vulnerable individuals with pre-existing health conditions: thrombotic effects in individuals with pre-existing coronary heart disease and impaired lung function in asthma sufferers (American Conference of Governmental Industrial Hygienists (ACGIH), 2005). Chronic long-term exposure has implicated PAH's reactive metabolites, to have the ability to bind to proteins and DNA, and exert carcinogenic effects (Bach et al. 2003). Such biochemical disruption and cellular damage has been most extensively researched in occupational studies, whereby high exposure has been associated with increased incidence of lung, bladder, skin, and gastrointestinal cancer (Boffetta et al. 1997) (Diggs et al. 2012) (Bach et al. 2003) (Olsson et al. 2010). Additionally, decreased immune function, cataracts, kidney and liver damage, including jaundice, have also been associated with high exposure (Kuo et al. 2012) (U.S. Department of health and human services, 1995). Whilst extensive research exists surrounding PAH's genotoxic and carcinogenic properties, an emerging body of evidence concerns PAH's neurotoxic effect through the induction of oxidative stress, inflammation (Saunders et al. 2006) and vascular injury within the brain (Hartz et al. 2008). Recently, research has emerged associating PAH exposure with impaired cognitive function and increased risk of neurodegeneration. 
To the best of our knowledge, from the large body of literature on the influence of air pollution in human health, the implications of $\mathrm{PAH}$ exposure specifically, on cognitive function and neurodegeneration in humans have not been systematically reviewed. Prior reviews have addressed the implications of PAH exposure on general health (Abdel-Shafy \& Mansour, 2016; Kiim et al. 2013), and its carcinogenic outcomes (Armstrong et al. 2004 ) (Bosetti, et al., 2007). The reviews which have made cognitive function and neurodegeneration the outcome of interest, include exposures to a vast mixture of air pollutants (Peters et al. 2019) (Schikowski \& Altug, 2020) (Kilian \& Kitazawa, 2018) (Calderón-Garcidueñas et al. 2016). In doing so we aim to disentangle the unique neurotoxic effect of PAH's from other pollutants in specific age groups and cognitiverelated functions to provide evidence for cognitive research and more vigilant monitoring and tighter restrictions on the main sources of emission. The Department for Environment, Food and Rural Affairs, currently considers annual monitoring of concentrations of one $\mathrm{PAH}$, Benzo[a]pyrene $(\mathrm{B}[\mathrm{a}] \mathrm{P})$, to be a sufficient representation of all atmospheric PAHs, and classifies the potential effect to human health of PAHs collectively, as 6 compounds, categorised as probably or possibly carcinogenic. No mention is made of the adverse neurological impact (Department for Environment, Food and Rural Affairs, 2011). A possible explanation is the consideration of concentration levels that constitute a risk for cancer, below which the effect of these pollutants can pass inadvertently. The UK national air quality objective for B[a]P is: $0.25 n g . \mathrm{m}^{-3}$. However, emissions of $\mathrm{B}[\mathrm{a}] \mathrm{P}$ have been increasing since 2008, and exceed this limit in multiple locations at multiple time points (Department or Environment, Food and Rural Affairs, 2020). Atmospheric PAH concentrations are subject to seasonal variation and climate (Finardi S, 2017). A further aim is to explore the influence the difference in PAH concentration in rural vs. metropolitan areas, can have, on outcomes involving cognitive function and neurodegeneration, to inform further studies.

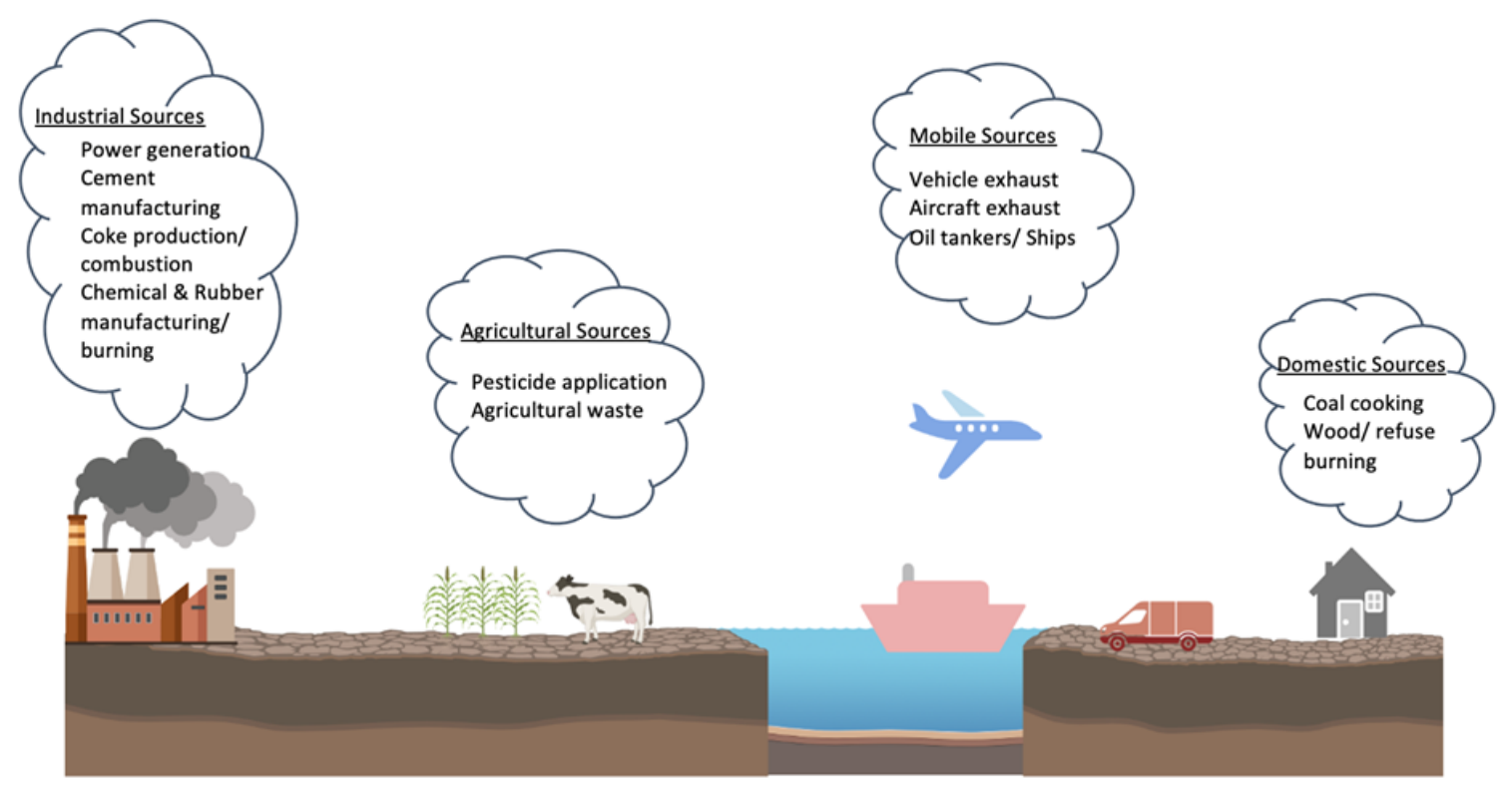


Figure 1: Anthropogenic sources (Industrial, Mobile, Domestic and Agricultural

Sources) of PAH release into the environment (figure made using: biorender.com).

\section{Methods}

\section{Eligibility criteria}

This review was conducted in line with the PRISMA guidelines (Liberati et al. 2009). Studies included were observational cohort studies of both male and female humans. Time of exposure was inclusive of the gestational period and stretched throughout life until death. Exposure quantification was limited to studies which measured the level of exposure to ambient PAHs, or PM2.5 through environmental air sampling or spatiotemporal modelling. Measures of exposure also included concentration of PAH metabolites in urine and dosimetry of PAH-DNA adducts, from DNA extracted from white blood cells. Outcomes included involved a formal assessment of outcomes of cognitive function, neurobehavioral symptoms of impaired cognition, and pathologies associated with neurodegeneration. Reports were limited to published scientific articles written in the English language. No publication dates were imposed. Studies were excluded if they did not fulfil the inclusion criteria, were not in humans, or where $\mathrm{PAH}$ exposure was measured as a component of diet, Environmental Tobacco Smoke (ETS) or Traffic Related Air Pollution (TRAP). Exposure via diet and ETS is not an appropriate representation of a major source of atmospheric $\mathrm{PAH}$. Prior research has elucidated contaminating pollutants within TRAP composition detrimentally affecting cognitive function. Inclusion of such studies would confound results and prevent us from elucidating the specific impact of $\mathrm{PAH}$ on cognition.

\section{$\underline{\text { Information Sources }}$}

Studies were identified by searching electronic databases: PubMed (1984- present) and Web of Science (1979- present). The last search was run on the $15^{\text {th }}$ February 2021. "Polycyclic Aromatic Hydrocarbons" in addition to the following search terms: "brain", "neurological", "cognitive", "cognition", "neurodegenerative", "neurodegeneration", "neurodevelopment", "neurodevelopmental" were used to identify articles in both databases. Limitations applied to the search included the fields searched being: Title and Abstract. In Web of Science the document type of: Articles was applied. In PubMed an additional limitation of the species selected being: Humans, was applied. Eligibility assessment was performed independently in an unbiased standardized manner by one reviewer. Ambiguity concerning the inclusion or exclusion of a study was resolved by a second 
reviewer being consulted and a consensus taken. Initial screening was performed through reviewing the Title and Abstract, after which, the full manuscript was reviewed.

\section{Data Collection process}

A data extraction sheet was developed and pilot tested on 5 randomly selected included studies, before being refined accordingly. One review author extracted data from the included studies, a second was consulted where ambiguity arose surrounding the appropriate data to extract. One author was contacted via email to provide numerical data that had only been presented graphically. Information extracted from studies included: sample size, sample characteristics, gender ratio, mean age, age range, comorbidities, air pollution component, time of exposure, air pollution data acquisition method and outcome measured.

\section{$\underline{\text { Risk of bias in individual studies }}$}

Risk of bias was assessed in line with the QADAS guidelines (University of Bristol, 2003). To ascertain the risk of bias within each study included, one reviewer working independently extracted the following information: participant inclusion/exclusion criteria explained, participant withdrawals from study explained, use of /comparison with a control/low exposure population, confounding variables identified, appropriate method/ analysis to adjust for confounding variables, outcome assessors aware of exposure status of study participant, intermediate or unexpected results explained/reported, and whether or not the methods of the study were reproducible.

\section{Planned methods of analysis}

Studies included were divided into 4 subgroups depending on the time at which the exposure was measured: prenatal, childhood, adult and finally childhood+adult. The category childhood+adult included studies whereby the cohort of people included was a mixture of individuals exposed only up until childhood, and others where exposure extended through to adulthood. Subsequently, studies were further divided into categories depending on the outcome measured: cognitive development, neurobehavioral development, general cognition and neurodegeneration. This subgroup division was conducted to adjust for heterogeneity between studies. The meta-analysis was performed by calculating odds ratios and $95 \%$ confidence for the effect sizes of reported outcomes. Forest plots were used to visualise differences in effect sizes between studies within the same subgroup. 


\section{$\underline{\text { Results }}$}

\section{$\underline{\text { Study Selection }}$}

The search of Web of Science and PubMed provided a total of 490 citations. After adjusting for duplicates 428 remained. Subsequent screening of the title and abstract resulted in a further 289 being discarded. Of the remaining 139, a further 102 were excluded upon further examination of the full manuscript and application of inclusion criteria. 1 reported data copied directly from a previous study (Tang et al. 2014). 16 were reviews, and did not include any primary data, 47 reported outcomes not relevant to cognitive function or neurodegeneration and in 38 the recorded exposure to PAH was not in keeping with the specified criteria. Resulting in a total of 37 studies included in this review. Subgroup division resulted in 21 prenatal exposure studies, 15 concerning cognitive development outcomes and 13 neurobehavioral development, 7 included measures of both. 11 childhood exposure studies, 3 general cognition, 5 neurobehavioral development and 3 neurodegeneration. 5 adult exposure studies all with outcomes of general cognition, and 3 childhood + adult studies all with measures of neurodegeneration. 1 study included measured outcomes for both prenatal and childhood exposure (Kerin et al. 2017) and 2 studies involved 2 different study cohorts one exposed only during childhood and the other included a mix of childhood and adult exposed subjects (Calderón-Garcidueñas et al. 2016; Calderón-Garcidueñas et al. 2018). Figure 2 depicts the flow chart for study inclusion and subgroup division. The full dataset can be found at the following link: https://doi.org/10.7488/ds/3031 .

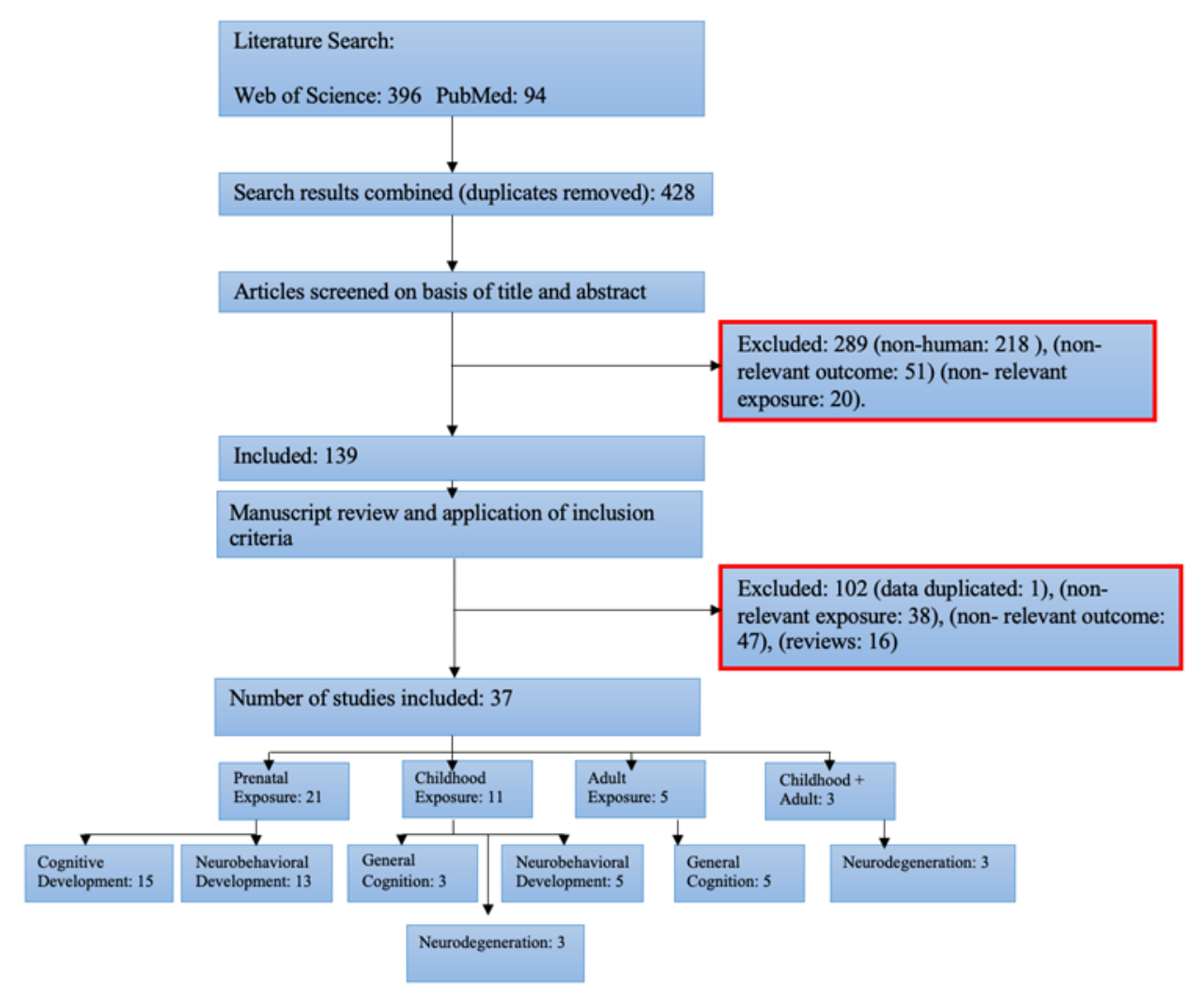


Figure 2: Flow chart of the search, study inclusion and subgroup division.

\section{Study Characteristics}

The 37 studies, involved populations from 9 countries (figure 3). Study population characteristics including sample size and mean age $( \pm S D)$ are displayed in figure 4.

11 included cohorts from the USA. 6 selected participants from the Columbia Centre for Children's Environmental Health cohort, however each selected different subgroups of the population, and measured different outcomes (Perera et al. 2006; Perera et al. 2018; Perera et al. 2015; Perera et al. 2012; Perera et al. 2011; Pagliaccio et al. 2020). 2 studies selected a subgroup of participants from the National Health and Nutrition Examination Survey 2001-2002 (NHANES) (Best et al. 2016), 1 of which included additional participants from the NHANES 2003-2004 cohort (Abid et al. 2014). The remaining 3 studies involved cohorts from the Childhood Autism Risks from Genetics and the Environment Study (Kerin et al. 2017), the Adolescent Brain Cognitive Development Study (Cserbik et al. 2020), and the Asthma Coalition on Community, Environment and Social Stress project (Chiu et al. 2016).

8 studies reported results from populations in China. 1 study involved a Taiyuan population (Niu et al. 2009) in addition to 2 selecting different subgroups from the Taiyuan Mother and Child Cohort Study (Cao et al. 2020; Nie et al. 2019). 3 involved populations from Tongliang (Perera et al. 2008; Tang et al. 2008; Tang et al. 2014), and the remaining 2 from Shanxi province (Du et al. 2020) and Qingdao City (Zhang et al. 2019).

5 studies involved populations from Spain. 2 involved a subgroup from the INfancia y Medio Ambiente Project (Lertxundi et al. 2015; Lertxundi et al. 2019) and the additional 3 participants from the Brian Development and Air Pollution Ultrafine Particles in School Children project (Mortamais et al. 2017; Rivas et al. 2019; Alemany et al. 2018).

4 studies reported on populations in Poland. 3 including participants from the Krakow Study (Jedrychowski et al. 2015; Edwards et al. 2010; Perera et al. 2013) and 1, the Polish Mother and Child Cohort Study (Polanska et al. 2013).

4 studies reported on populations in Mexico. All involved Mexico City residents (CalderónGarcidueñas et al. 2020), and 1 of 6 (Calderón-Garcidueñas et al. 2015; Calderón-Garcidueñas et al. 2016), or 3 small Mexican cities (Calderón-Garcidueñas et al. 2018) respectively.

2 studies involved a Korean population (Cho et al. 2020; Ha et al. 2012). Further individual studies included populations from the Czech Republic (Blazkova et al. 2020), Kenya (Suter et al. 2017) and Belgium (Saenen et al. 2016). 


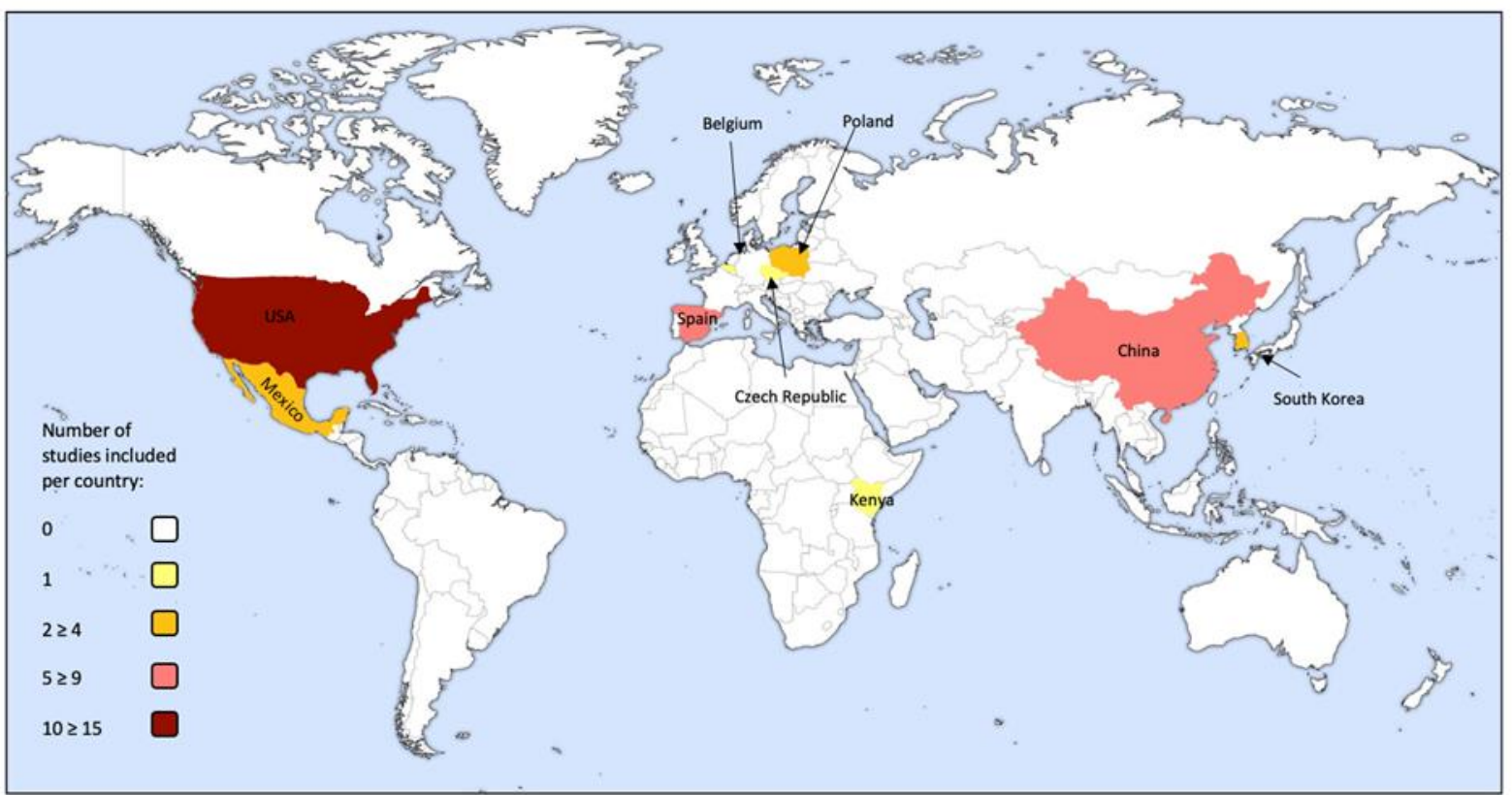

Figure 3: Global Distribution of the population cohorts in each of the 37 studies included in this review (figure made using: biorender.com). 


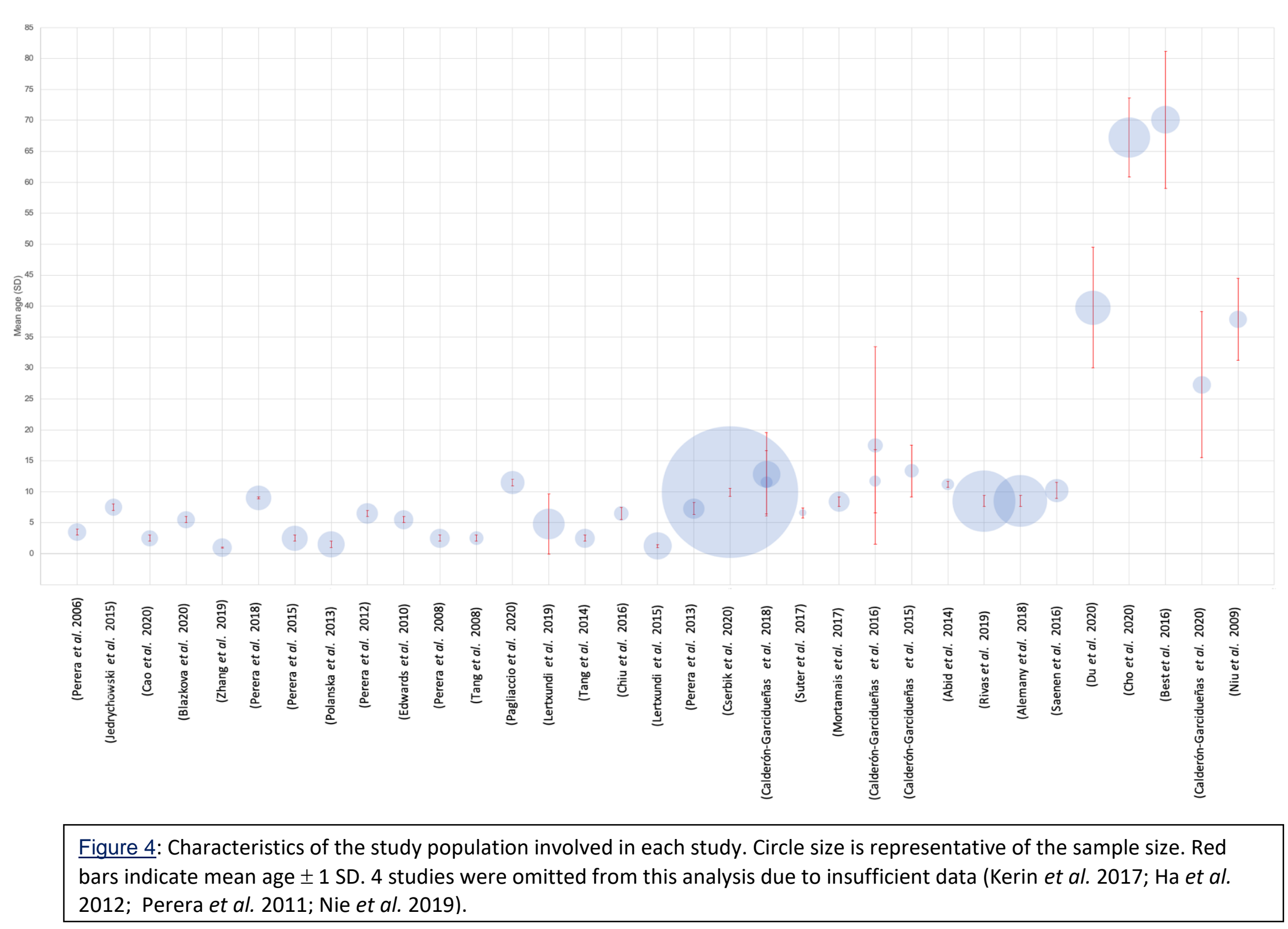

bars indicate mean age \pm 1 SD. 4 studies were omitted from this analysis due to insufficient data (Kerin et al. 2017; Ha et al.

012; Perera et al. 2011; Nie et al. 2019). 


\section{Exposure assessment}

Of the 37 studies included, 7 measured exposure through environmental PAH sampling, 5 by environmental $\mathrm{PM}_{2.5}$ sampling, 7 by $\mathrm{PM}_{2.5}$ spatiotemporal modelling, 10 by concentrations of $\mathrm{PAH}$ metabolites in urine and 8 using dosimetry to measure PAH-DNA adducts (figure 5).

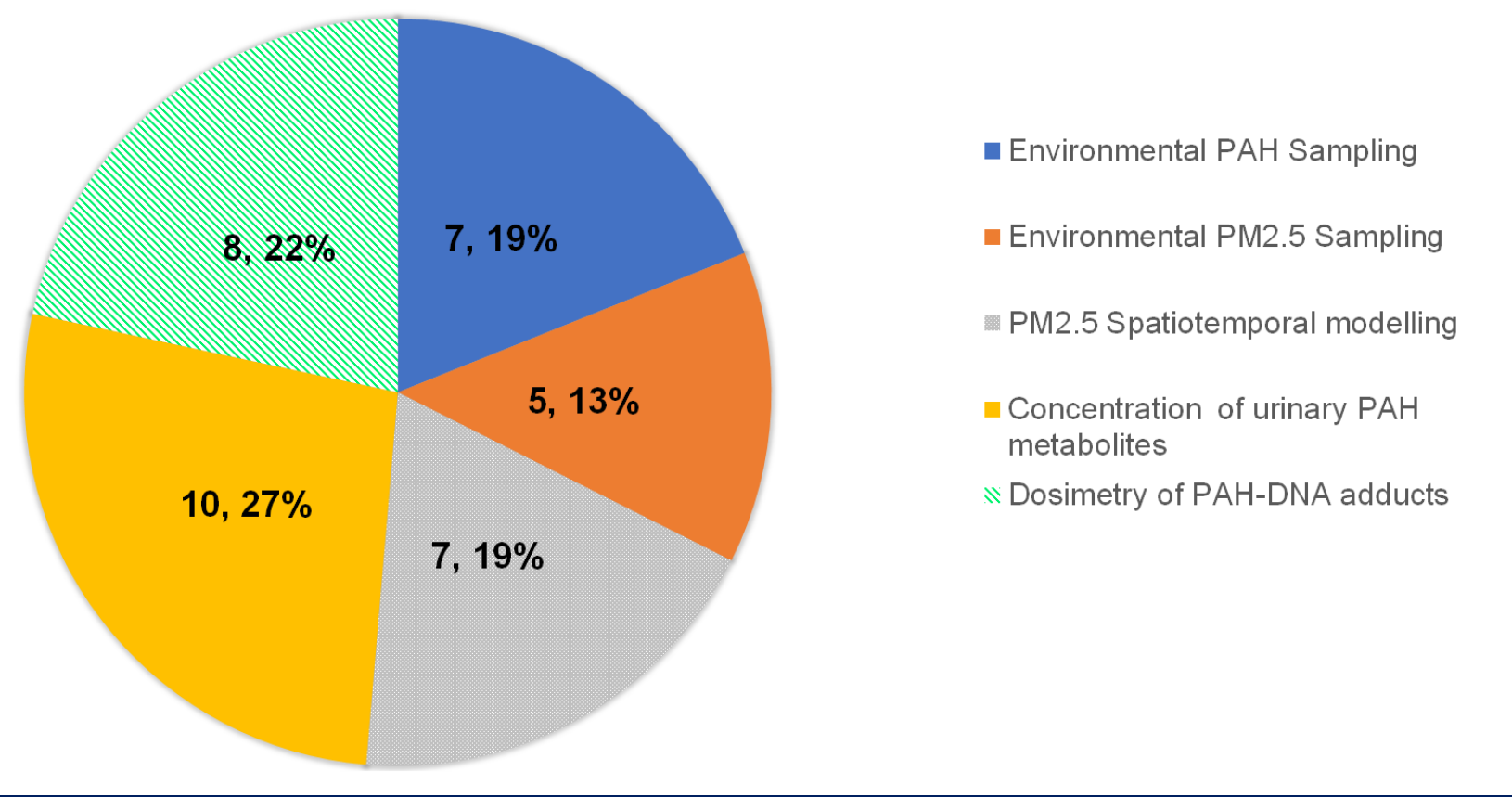

Figure 5: Pie chart representing the proportion of included studies measuring exposure to PAH as a measure of: environmental PAH Sampling, environmental $\mathrm{PM}_{2.5}$ sampling, $\mathrm{PM}_{2.5}$ spatiotemporal modelling, concentration of urinary $\mathrm{PAH}$ metabolites and dosimetry of PAH-DNA adducts.

\section{$\underline{\text { Outcome assessment }}$}

Outcomes included 21 different tests measuring cognitive function, 9 different tests measuring neurobehavioral symptoms of impaired cognition and 3 different measures of pathologies associated with neurodegeneration (figure 6). 


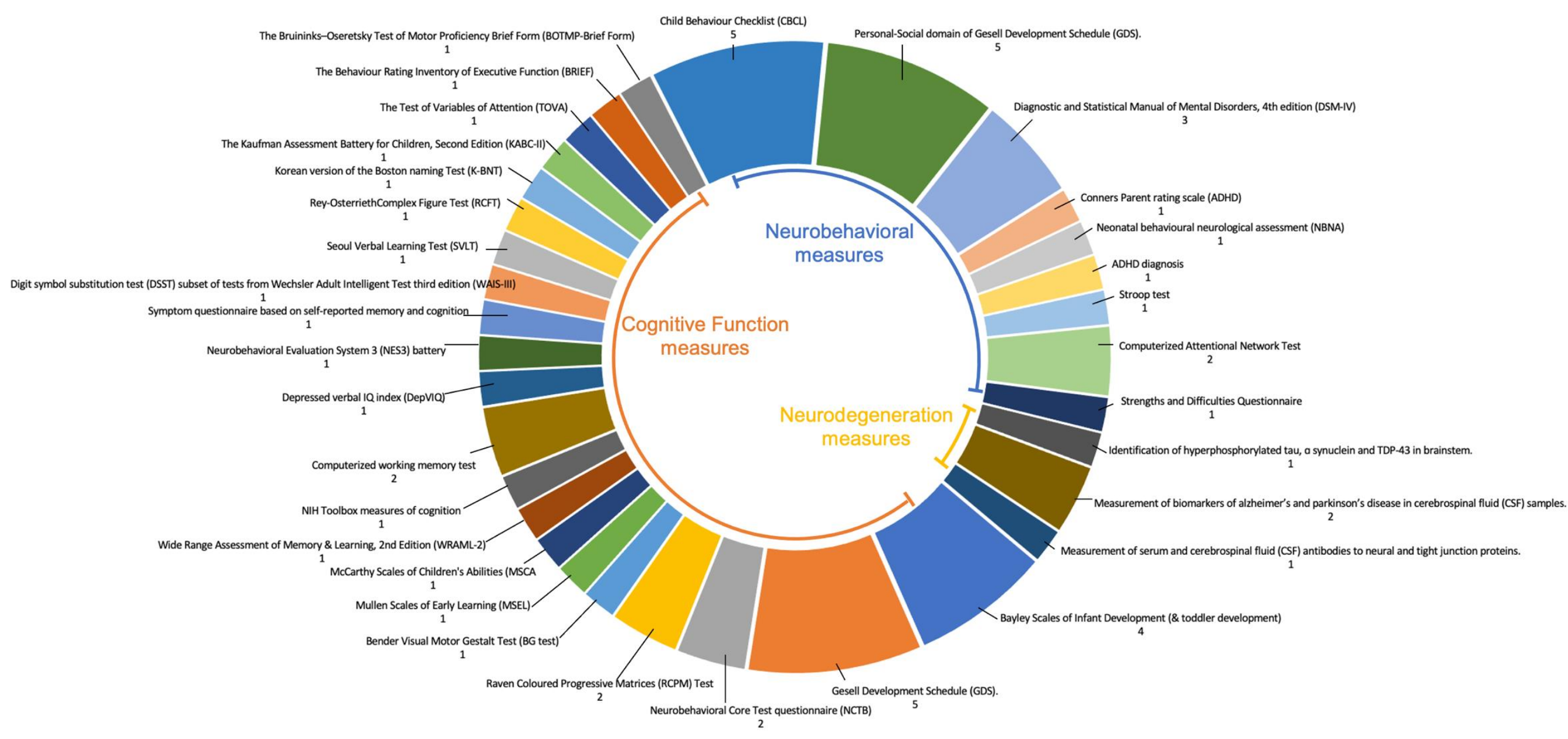

Figure 6: Pie chart representing the number of studies using different tests to measure outcomes. Measures include cognitive function,

neurobehavioral symptoms of impaired cognition, and pathologies associated with neurodegeneration. 


\section{Prenatal Exposure}

\section{$\underline{\text { Association between prenatal PAH exposure and cognitive development }}$}

Children with a high prenatal PAH exposure were found to have a delay in overall child intelligence $(\mathrm{OR}=1.75,95 \% \mathrm{Cl}, 1.11$ to 2.71$)$ (Edwards et al. 2010), mental development $(\mathrm{OR}=0.65$, Perera et al. 2015) and average overall development (Perera et al. 2008; Tang et al. 2014) (OR= 0.84, 95\% $\mathrm{Cl}, 0.52$ to $1.36 ; \mathrm{OR}=1.85,95 \% \mathrm{Cl}, 1.13$ to 3.01 respectively). Specifically, the greatest negative effects reported were in verbal IQ (OR=3.45, 95\% $\mathrm{Cl}, 0.95$ to 12.49 ) (Jedrychowski et al. 2015) and language (OR=5.99, 95\%Cl, 1.88 to 19.02) (Zhang et al. 2015). However, the latter could not be confirmed in $5 / 6$ studies (Perera et al. 2008; Tang et al. 2008; Tang et al. 2014; Cao et al. 2020; Polanska et al. 2013). Two studies analysed the effect of $\mathrm{PAH}$ in general cognitive abilities with contradictory results: Perera et al (2006) reported a negative effect $(\mathrm{OR}=2.89,95 \% \mathrm{Cl}, 1.33$ to 6.25$)$ while Polanska et al (2013) reported no effect. PAH effect on impaired motor development was inconclusive, as it was confirmed by 4 studies (Perera et al. 2008; Tang et al. 2008; Tang et al. 2014; Cao et al. 2020) $(\mathrm{OR}=0.95,95 \% \mathrm{Cl}, 0.58$ to $1.53 ; \mathrm{OR}=1.91,95 \% \mathrm{Cl}, 1.22$ to $2.97 ; \mathrm{OR}=1.63$, $95 \% \mathrm{Cl}, 1.00$ to $2.65 ; \mathrm{OR}=1.82,95 \% \mathrm{Cl}, 3.21$ to 1.03 respectively) whereas 3 others could not confirm it. No association was found between $\mathrm{PAH}$ exposure and developmental motor ability by Polanska et al (2013), fine and gross motor abilities by Zhang et al (2019) and psychomotor abilities by Perera et al (2006).

Only one study reported effect of PAH and reduced adaptive development (Tang et al. 2014) $(\mathrm{OR}=1.77,95 \% \mathrm{Cl}, 1.09$ to 2.88$)$ while $4 / 5$ reported no association with adaptive domains (Zhang et al. 2019; Perera et al. 2008; Tang et al. 2008; Cao et al. 2020). Size effects reported by the studies mentioned are graphically represented in figure 7 and listed in table 1. 


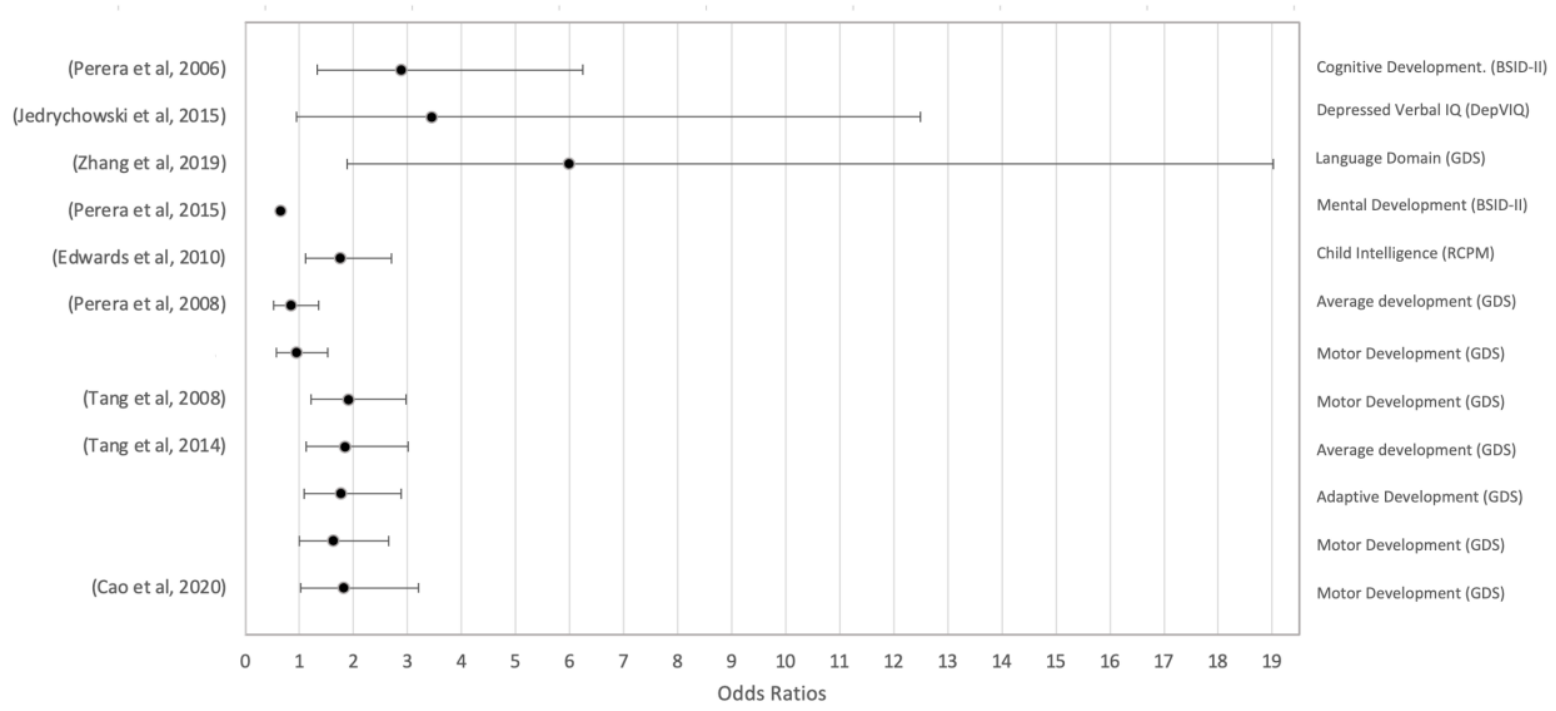

Figure 7 : Forest Plot of the calculated odds ratios (OR) and $95 \%$ confidence intervals $(95 \% \mathrm{Cl})$ for the association between prenatal exposure to PAH and measures of Cognitive Development. Bayley Scales of Infant Development- Revised (BSID-II), Depressed Verbal IQ Index (DepVIQ), Gesell Development Schedule (GDS), Raven Coloured Progressive Matrices Test (RCPM), Wechsler Intelligence Scale for Children IV (WISC-IV). 1 study had insufficient data to calculate $95 \% \mathrm{Cl}$ (Perera et al. 2015) 
Table 1: Studies with measured prenatal PAH exposure on cognitive development.

\begin{tabular}{|c|c|c|c|c|c|c|c|}
\hline Citation & $\begin{array}{l}\text { Sample } \\
\text { Size }\end{array}$ & Sample Characteristics & $\begin{array}{l}\text { Male: } \\
\text { Female }\end{array}$ & $\begin{array}{l}\text { Mean } \\
\text { age } \\
\text { (SD) }\end{array}$ & $\begin{array}{l}\text { Age } \\
\text { Range }\end{array}$ & $\begin{array}{l}\text { Comorbidi } \\
\text { ties }\end{array}$ & $\begin{array}{l}\text { Air Pollution Data } \\
\text { Acquisition Method }\end{array}$ \\
\hline $\begin{array}{l}\text { Perera et } \\
\text { al. } 2006\end{array}$ & 183 & $\begin{array}{l}\text { Children } 3 \text { years of age, mothers } 18-35 \text { years, non- } \\
\text { smoking, free of diabetes, hypertension, or known } \\
\text { HIV, African American and Dominican women } \\
\text { residing for a minimum of a year in Washington } \\
\text { Heights, Harlem or the South Bronx in New York City }\end{array}$ & $84: 99$ & $3.5(0.5)$ & $\begin{array}{l}3 \text { years - } \\
3 \text { years } \\
12 \\
\text { months }\end{array}$ & $\mathrm{N} / \mathrm{A}$ & $\begin{array}{l}\text { Environmental } \\
\text { samples analysed } \\
\text { for } 8 \text { PAHs }\end{array}$ \\
\hline $\begin{array}{l}\text { Jedrycho } \\
\text { wski et al. } \\
2015\end{array}$ & 170 & $\begin{array}{l}\text { Children } 7 \text { years of age, mothers } \geq 18 \text { years of age, } \\
\text { non-smoking, singleton pregnancies, no history of } \\
\text { illicit drug use, pregnancy related diabetes, or } \\
\text { hypertension, no current occupational exposure to } \\
\text { PAH or any other known developmental toxicants, } \\
\text { and have been resident in Krakow, Poland for a } \\
\text { minimum of a year }\end{array}$ & $80: 90$ & $7.5(0.5)$ & $\begin{array}{l}7 \text { years - } \\
7 \text { years } \\
12 \text { months }\end{array}$ & $\mathrm{N} / \mathrm{A}$ & $\begin{array}{l}\text { Cord blood PAH- } \\
\text { DNA adduct }\end{array}$ \\
\hline $\begin{array}{l}\text { Zhang et } \\
\text { al. } 2019\end{array}$ & 211 & $\begin{array}{l}\text { Infants } 12 \text { months of age, free from delivery injuries, } \\
\text { neonatal problems, acquired disabilities, } \\
\text { developmental dysplasia or other developmental } \\
\text { defects, Mothers resident in Qingdao city, China for } \\
\text { at least } 3 \text { years, free from diabetes, known HIV and } \\
\text { known neuropsychiatric disease. }\end{array}$ & $192: 156$ & $\begin{array}{l}1.0 \\
(0.083)\end{array}$ & $\begin{array}{l}1 \text { year - } 1 \\
\text { year } 1 \\
\text { month }\end{array}$ & $\mathrm{N} / \mathrm{A}$ & $\begin{array}{l}\text { Cord blood } \\
\text { Benzo[a]pyrene- } \\
\text { DNA adducts } \\
\text { (ng/mL) }\end{array}$ \\
\hline $\begin{array}{l}\text { Perera et } \\
\text { al. } 2015\end{array}$ & 380 & $\begin{array}{l}\text { Children } 2 \text { years of age, mothers } 18-35 \text { years, non- } \\
\text { smoking, free of diabetes, hypertension, or known } \\
\text { HIV, African American and Dominican women } \\
\text { residing for a minimum of a year in Washington } \\
\text { Heights, Harlem or the South Bronx in New York City. }\end{array}$ & $\mathrm{N} / \mathrm{A}$ & $2.5(0.5)$ & $\begin{array}{l}2 \text { years - } \\
2 \text { years } \\
12 \\
\text { months }\end{array}$ & $\mathrm{N} / \mathrm{A}$ & $\begin{array}{l}\text { PAH/aromatic DNA } \\
\text { adducts in umbilical } \\
\text { cord blood samples }\end{array}$ \\
\hline $\begin{array}{l}\text { Polanska } \\
\text { et al. } \\
2013\end{array}$ & 406 & $\begin{array}{l}\text { Children 1-2 years of age, mothers had single } \\
\text { pregnancy up to } 12 \text { weeks of gestation, no assisted } \\
\text { conception, no pregnancy complications, no chronic } \\
\text { disease, resident in Poland }\end{array}$ & $192: 214$ & $1.5(0.5)$ & $\begin{array}{l}1 \text { year }-2 \\
\text { years } 12 \\
\text { months }\end{array}$ & $\mathrm{N} / \mathrm{A}$ & $\begin{array}{l}\text { 1-hydroxypyrene } \\
\text { metabolites in } \\
\text { mothers urine }\end{array}$ \\
\hline
\end{tabular}




\begin{tabular}{|c|c|c|c|c|c|c|c|}
\hline $\begin{array}{l}\text { Edwards } \\
\text { et al. } \\
2010\end{array}$ & 214 & $\begin{array}{l}\text { Children } 5 \text { years of age, mothers } \geq 18 \text { years of age, } \\
\text { non-smoking, singleton pregnancies, no history of } \\
\text { illicit drug use, pregnancy related diabetes, or } \\
\text { hypertension, no current occupational exposure to } \\
\text { PAH or any other known developmental toxicants, } \\
\text { and have been resident in Krakow, Poland for a } \\
\text { minimum of a year }\end{array}$ & 103:111 & $5.5(0.5)$ & $\begin{array}{l}5 \text { years - } \\
5 \text { years } \\
12 \\
\text { months }\end{array}$ & $N / A$ & $\begin{array}{l}\text { Environmental } \\
\text { samples analysed } \\
\text { for } 8 \text { PAHs }\end{array}$ \\
\hline $\begin{array}{l}\text { Perera et } \\
\text { al. } 2008\end{array}$ & 217 & $\begin{array}{l}\text { Children } 2 \text { years of age, born between either: March- } \\
\text { June } 2002 \text { or March- May } 2002 \text {, mothers } \geq 20 \text { years, } \\
\text { non-smoking, resident within } 2 \mathrm{~km} \text { of Tongliang power } \\
\text { plant }\end{array}$ & 113: 104 & $2.5(0.5)$ & $\begin{array}{l}2 \text { years - } \\
2 \text { years } \\
12 \\
\text { months }\end{array}$ & $N / A$ & $\begin{array}{l}\text { Cord blood } \\
\text { Benzo[a]pyrene- } \\
\text { DNA adducts } \\
\text { (ng/mL) }\end{array}$ \\
\hline $\begin{array}{l}\text { Tang et } \\
\text { al. } 2008\end{array}$ & 110 & $\begin{array}{l}\text { Children } 2 \text { years of age, born between March- June } \\
2002 \text {, Mothers } \geq 20 \text { years, non-smoking, resident } \\
\text { within } 2 \mathrm{~km} \text { of Tongliang power plant }\end{array}$ & 54:56 & $2.5(0.5)$ & $\begin{array}{l}2 \text { years - } \\
2 \text { years } \\
12 \\
\text { months }\end{array}$ & N/A & $\begin{array}{l}\text { Cord blood } \\
\text { Benzo[a]pyrene- } \\
\text { DNA adducts } \\
\text { (ng/mL) }\end{array}$ \\
\hline $\begin{array}{l}\text { Tang et } \\
\text { al. } 2014\end{array}$ & 215 & $\begin{array}{l}\text { Children } 2 \text { years of age, born between either: March- } \\
\text { June } 2002 \text { or March- May } 2002 \text {, mothers } \geq 20 \text { years, } \\
\text { non-smoking, resident within } 2 \mathrm{~km} \text { of Tongliang power } \\
\text { plant }\end{array}$ & 106:109 & $2.5(0.5)$ & $\begin{array}{l}2 \text { years - } \\
2 \text { years } \\
12 \\
\text { months }\end{array}$ & N/A & $\begin{array}{l}\text { Cord blood } \\
\text { Benzo[a]pyrene- } \\
\text { DNA adducts } \\
(\mathrm{ng} / \mathrm{mL})\end{array}$ \\
\hline $\begin{array}{l}\text { Cao et al. } \\
2020\end{array}$ & 158 & $\begin{array}{l}\text { Children } 2 \text { years of age, mothers } \geq 18 \text { years of age, } \\
\text { non-smoking, resident in Taiyuan, Shanxi province, } \\
\text { China for a minimum of } 1 \text { year }\end{array}$ & $82: 76$ & $2.5(0.5)$ & $\begin{array}{l}2 \text { years - } \\
2 \text { years } \\
12 \\
\text { months }\end{array}$ & N/A & $\begin{array}{l}\text { The sum of the } \\
\text { maternal } \\
\text { concentrations of } \\
\text { eleven urinary PAHs } \\
\text { metabolites } \Sigma \text { - } \\
\text { OHPAHs }\end{array}$ \\
\hline
\end{tabular}




\section{$\underline{\text { Association between prenatal PAH exposure and neurobehavioral development }}$}

Children with a high prenatal PAH exposure were found to exhibit externalizing and internalizing behavioural problems $(\mathrm{OR}=2.49,95 \% \mathrm{Cl}, 1.57$ to $3.95 ; \mathrm{OR}=2.39,95 \% \mathrm{Cl}, 1.51$ to 3.79 respectively) (Perera et al. 2013) and infants exhibited a decrease in behavioural development (OR=2,95\%Cl, 1.27 to 3.15) (Nie et al. 2019). Associations with anxious/ depressed behaviour were found in 3/4 studies (Perera et al. 2012; Perera et al. 2011; Perera et al. 2013) (OR=8.89, 95\%Cl, 1.7 to 46.51 ; $\mathrm{OR}=8.14,95 \% \mathrm{Cl}, 1.21$ to 54.94 ; $\mathrm{OR}=1.7,95 \% \mathrm{Cl}, 1.08$ to 2.68 respectively) with no association found by Pagliaccio et al (2020). 3/5 studies reported negative effect on child attentiveness (Perera et al. 2018; Pagliaccio et al. 2020; Perera et al. 2012) (OR=1.34, 95\%, 0.85 to 1.83; OR=2.02, 95\%, 1.35 to $3.03 ; \mathrm{OR}=3.79,95 \% \mathrm{Cl}, 1.14$ to 12.66$)$ whilst Perera et al (2011) and Perera et al (2013) reported no effect. Perera et al (2013) report of an effect in both withdrawn/ depressed and aggressive behaviour $(\mathrm{OR}=2,95 \% \mathrm{Cl}, 1.27$ to 3.16 ; $\mathrm{OR}=2.29,95 \% \mathrm{Cl}, 1.45$ to 3.62 respectively) was contradicted by Pagliaccio et al (2020) reporting no effect for either. Pagliaccio et al (2020) did however report an effect on impaired thought problems (OR= $1.95,95 \% \mathrm{Cl}, 1.3$ to 2.91 ) which was contradicted by Perera et al (2013). Only $1 / 7$ studies reported an association between PAH and social problems (Perera et al. 2013) (OR=1.57, $95 \% \mathrm{Cl}, 1.00$ to 2.48), the remaining 6 reported no effect (Pagliaccio et al. 2020; Zhang et al. 2019, Perera et al. 2008; Tang et al. 2008; Tang et al. 2014; Cao et al. 2020). Both Perera et al (2013) and Pagliaccio et al (2020) found no effect on rule breaking behaviour or somatic complaints. Perera et al (2018) reported no associations with attention deficit hyperactivity disorder (ADHD) index scores, or hyperactive compulsive behaviour nor did Perera et al (2006) with total behavioural problems. Studies reporting neurobehavioral effects are reported in table 2 , and effect sizes depicted in figure 8 . 


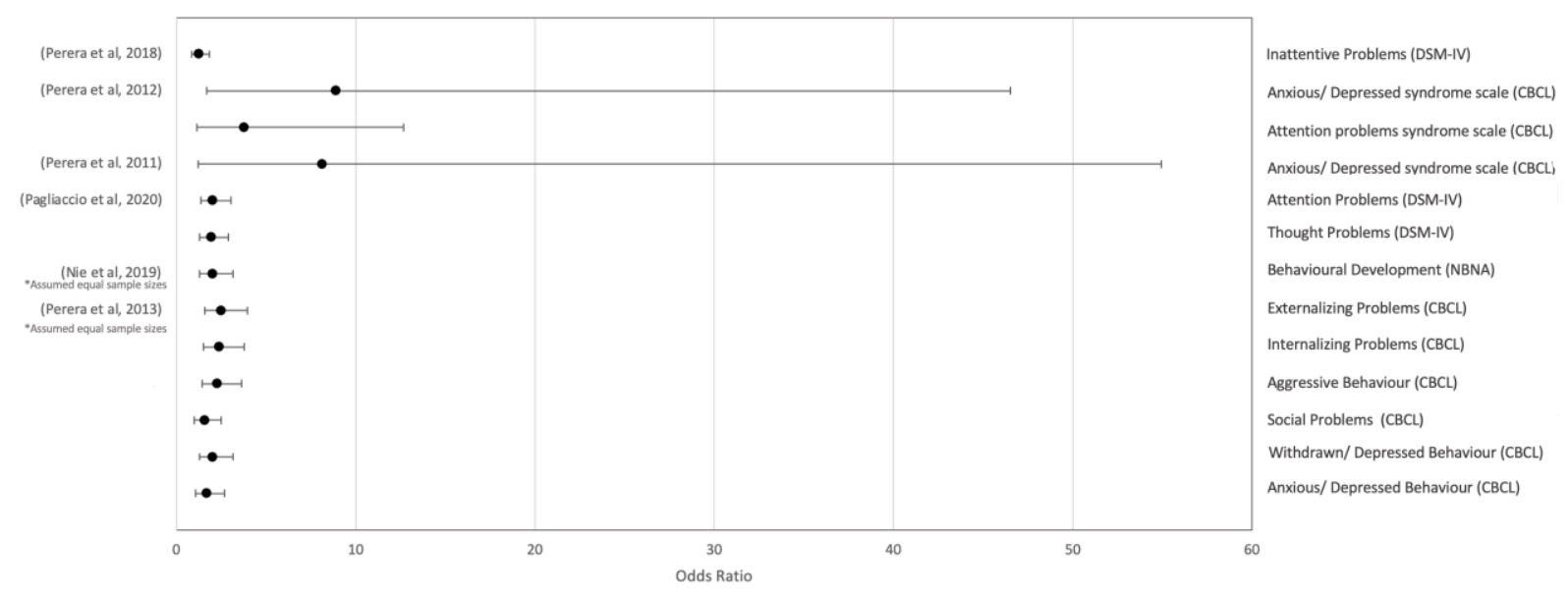

Figure 8: A Forest Plot of the calculated odds ratios (OR) and 95\% confidence intervals $(95 \% \mathrm{Cl})$ for the association between prenatal exposure to $\mathrm{PAH}$ and measures of Neurobehavioral Development. Gesell Development Schedule, Diagnostic (GDS) and Statistical Manual of Mental Disorders, 4th Edition (DSM-IV), Child Behaviour Checklist (CBCL), Neonatal Behavioural Neurological Assessment (NBNA). *sample sizes were assumed equal 
Table 2: Studies with measured prenatal PAH exposure on neurobehavioral development.

\begin{tabular}{|c|c|c|c|c|c|c|c|}
\hline Citation & $\begin{array}{l}\text { Sample } \\
\text { Size }\end{array}$ & Sample Characteristics & $\begin{array}{l}\text { Male: } \\
\text { Female }\end{array}$ & $\begin{array}{l}\text { Mean } \\
\text { age } \\
\text { (SD) }\end{array}$ & $\begin{array}{l}\text { Age } \\
\text { Range }\end{array}$ & $\begin{array}{l}\text { Comorbi } \\
\text { dities }\end{array}$ & $\begin{array}{l}\text { Air Pollution Data } \\
\text { Acquisition } \\
\text { Method }\end{array}$ \\
\hline $\begin{array}{l}\text { Perera et } \\
\text { al. } 2018\end{array}$ & 351 & $\begin{array}{l}\text { Children } 9 \text { years of age, mothers } 18-35 \text { years, non- } \\
\text { smoking, free of diabetes, hypertension, or known HIV, } \\
\text { African American and Dominican women residing for a } \\
\text { minimum of a year in Washington Heights, Harlem or the } \\
\text { South Bronx in New York City. }\end{array}$ & 163: 188 & $\begin{array}{l}9.01 \\
(0.19)\end{array}$ & $\begin{array}{l}9 \text { years- } 9 \\
\text { years } 12 \\
\text { months }\end{array}$ & $\mathrm{N} / \mathrm{A}$ & $\begin{array}{l}\text { Cord blood } \\
\text { Benzo[a]pyrene- } \\
\text { DNA adducts } \\
\text { (ng/mL) }\end{array}$ \\
\hline $\begin{array}{l}\text { Perera et } \\
\text { al. } 2012\end{array}$ & 253 & $\begin{array}{l}\text { Children 6-7 years of age, mothers } 18-35 \text { years, non- } \\
\text { smoking, free of diabetes, hypertension, or known HIV, } \\
\text { African American and Dominican women residing for a } \\
\text { minimum of a year in Washington Heights, Harlem or the } \\
\text { South Bronx in New York City. }\end{array}$ & $131: 122$ & $\begin{array}{l}6.5 \\
(0.5)\end{array}$ & $\begin{array}{l}6 \text { years - } \\
7 \text { years } \\
12 \\
\text { months }\end{array}$ & $\mathrm{N} / \mathrm{A}$ & $\begin{array}{l}\text { Environmental } \\
\text { samples analysed } \\
\text { for } 8 \text { PAHs }\end{array}$ \\
\hline $\begin{array}{l}\text { Perera et } \\
\text { al. } 2011\end{array}$ & 215 & $\begin{array}{l}\text { Children } 3 \text { years } 9 \text { months- } 5 \text { years } 11 \text { months of age, } \\
\text { mothers } 18-35 \text { years, non-smoking, free of diabetes, } \\
\text { hypertension, or known HIV, African American and } \\
\text { Dominican women residing for a minimum of a year in } \\
\text { Washington Heights, Harlem or the South Bronx in New } \\
\text { York City. }\end{array}$ & 87: 128 & $\begin{array}{l}4.8 \\
\text { (not } \\
\text { reporte } \\
\text { d) }\end{array}$ & $\begin{array}{l}3 \text { years } 9 \\
\text { months - } \\
5 \text { years } \\
11 \\
\text { months }\end{array}$ & $\mathrm{N} / \mathrm{A}$ & $\begin{array}{l}\text { Cord blood } \\
\text { Benzo[a]pyrene- } \\
\text { DNA adducts } \\
\text { (ng/mL) }\end{array}$ \\
\hline $\begin{array}{l}\text { Pagliacci } \\
\text { o et al. } \\
2020\end{array}$ & 319 & $\begin{array}{l}\text { Children } 11 \text { years old, mothers } 18-35 \text { years, non-smoking, } \\
\text { free of diabetes, hypertension, or known HIV, African } \\
\text { American and Dominican women residing for a minimum } \\
\text { of a year in Washington Heights, Harlem or the South } \\
\text { Bronx in New York City. }\end{array}$ & 177: 142 & $\begin{array}{l}11.5 \\
(0.5)\end{array}$ & $\begin{array}{l}11 \text { years - } \\
11 \text { years } \\
12 \\
\text { months }\end{array}$ & $\begin{array}{l}\text { Early Life } \\
\text { Stress }\end{array}$ & $\begin{array}{l}\text { Environmental } \\
\text { samples analysed } \\
\text { for } 8 \text { PAHs }\end{array}$ \\
\hline $\begin{array}{l}\text { Nie et al. } \\
2019\end{array}$ & 247 & $\begin{array}{l}\text { Infants } 3 \text { days of age, mothers } \geq 18 \text { years, non-smoking, } \\
\text { no chronic disease or family history of neurological } \\
\text { disease, single gestational viable fetus, who delivered in } \\
\text { the Sixth Hospital of Shanxi Medical University and the } \\
\text { Eighth People's Hospital of Taiyua, resident in Taiyuan for } \\
\text { at least a year }\end{array}$ & 132: 115 & $\begin{array}{l}3 \text { days } \\
\text { (not } \\
\text { reporte } \\
\text { d) }\end{array}$ & 3 days & $\mathrm{N} / \mathrm{A}$ & $\begin{array}{l}\text { Urinary metabolite } \\
\text { concentrations of } \\
\text { 2-hydroxyfluorene }\end{array}$ \\
\hline
\end{tabular}




\begin{tabular}{|c|c|c|c|c|c|c|c|}
\hline $\begin{array}{l}\text { Perera et } \\
\text { al. } 2013\end{array}$ & 248 & $\begin{array}{l}\text { Children from Krakow, Poland, mothers } \geq 18 \text { years, non- } \\
\text { smoking }\end{array}$ & 122: 126 & $\begin{array}{l}7.28 \\
(0.98)\end{array}$ & $\begin{array}{l}6 \text { years - } \\
9 \text { years } \\
12 \\
\text { months }\end{array}$ & $\begin{array}{l}\text { Maternal } \\
\text { Psycholo } \\
\text { gical } \\
\text { Distress }\end{array}$ & $\begin{array}{l}\text { Personal air } \\
\text { monitoring } \\
\text { analysing } \\
\text { concentrations of } 8 \\
\text { PAHs }\end{array}$ \\
\hline $\begin{array}{l}\text { Tang et } \\
\text { al. } 2008\end{array}$ & 110 & $\begin{array}{l}\text { Children } 2 \text { years of age, born between March- June } 2002 \text {, } \\
\text { mothers } \geq 20 \text { years, non-smoking, resident within } 2 \mathrm{~km} \text { of } \\
\text { Tongliang power plant }\end{array}$ & $54: 56$ & $\begin{array}{l}2.5 \\
(0.5)\end{array}$ & $\begin{array}{l}2 \text { years - } \\
2 \text { years } \\
12 \\
\text { months }\end{array}$ & $\mathrm{N} / \mathrm{A}$ & $\begin{array}{l}\text { Cord blood } \\
\text { Benzo[a]pyrene- } \\
\text { DNA adducts } \\
(\mathrm{ng} / \mathrm{mL})\end{array}$ \\
\hline $\begin{array}{l}\text { Zhang et } \\
\text { al. } 2019\end{array}$ & 211 & $\begin{array}{l}\text { Infants } 12 \text { months of age, free from delivery injuries, } \\
\text { neonatal problems, acquired disabilities, developmental } \\
\text { dysplasia or other developmental defects, mothers } \\
\text { resident in Qingdao city, China for at least } 3 \text { years, free } \\
\text { from diabetes, known HIV and known neuropsychiatric } \\
\text { disease. }\end{array}$ & 192:156 & $\begin{array}{l}1.0 \\
(0.083)\end{array}$ & $\begin{array}{l}1 \text { year }-1 \\
\text { year } 1 \\
\text { month }\end{array}$ & $\mathrm{N} / \mathrm{A}$ & $\begin{array}{l}\text { Cord blood } \\
\text { Benzo[a]pyrene- } \\
\text { DNA adducts } \\
(\mathrm{ng} / \mathrm{mL})\end{array}$ \\
\hline $\begin{array}{l}\text { Perera et } \\
\text { al. } 2008\end{array}$ & 217 & $\begin{array}{l}\text { Children } 2 \text { years of age, born between either: March- June } \\
2002 \text { or March- May } 2002 \text {, mothers } \geq 20 \text { years, non- } \\
\text { smoking, resident within } 2 \mathrm{~km} \text { of Tongliang power plant }\end{array}$ & 113: 104 & $\begin{array}{l}2.5 \\
(0.5)\end{array}$ & $\begin{array}{l}2 \text { years - } \\
2 \text { years } \\
12 \\
\text { months }\end{array}$ & $\mathrm{N} / \mathrm{A}$ & $\begin{array}{l}\text { Cord blood } \\
\text { Benzo[a]pyrene- } \\
\text { DNA adducts } \\
\text { (ng/mL) }\end{array}$ \\
\hline $\begin{array}{l}\text { Tang et } \\
\text { al. } 2014\end{array}$ & 215 & $\begin{array}{l}\text { Children } 2 \text { years of age, born between either: March- June } \\
2002 \text { or March- May } 2002 \text {, mothers } \geq 20 \text { years, non- } \\
\text { smoking, resident within } 2 \mathrm{~km} \text { of Tongliang power plant }\end{array}$ & $106: 109$ & $\begin{array}{l}2.5 \\
(0.5)\end{array}$ & $\begin{array}{l}2 \text { years - } \\
2 \text { years } \\
12 \\
\text { months }\end{array}$ & $\mathrm{N} / \mathrm{A}$ & $\begin{array}{l}\text { Cord blood } \\
\text { Benzo[a]pyrene- } \\
\text { DNA adducts } \\
(\mathrm{ng} / \mathrm{mL})\end{array}$ \\
\hline $\begin{array}{l}\text { Cao et al. } \\
2020\end{array}$ & 158 & $\begin{array}{l}\text { Children } 2 \text { years of age, mothers } \geq 18 \text { years of age, non- } \\
\text { smoking, resident in Taiyuan, Shanxi province, China for a } \\
\text { minimum of } 1 \text { year }\end{array}$ & $82: 76$ & $\begin{array}{l}2.5 \\
(0.5)\end{array}$ & $\begin{array}{l}2 \text { years - } \\
2 \text { years } \\
12 \\
\text { months }\end{array}$ & $\mathrm{N} / \mathrm{A}$ & $\begin{array}{l}\text { The sum of the } \\
\text { maternal } \\
\text { concentrations of } \\
\text { eleven urinary } \\
\text { PAHs metabolites } \\
\Sigma \text {-OHPAHs }\end{array}$ \\
\hline $\begin{array}{l}\text { Perera et } \\
\text { al. } 2006\end{array}$ & 183 & $\begin{array}{l}\text { Children } 3 \text { years of age, mothers } 18-35 \text { years, non- } \\
\text { smoking, free of diabetes, hypertension, or known HIV, } \\
\text { African American and Dominican women residing for a } \\
\text { minimum of a year in Washington Heights, Harlem or the } \\
\text { South Bronx in New York City }\end{array}$ & $84: 99$ & $\begin{array}{l}3.5 \\
(0.5)\end{array}$ & $\begin{array}{l}3 \text { years - } \\
3 \text { years } \\
12 \\
\text { months }\end{array}$ & $\mathrm{N} / \mathrm{A}$ & $\begin{array}{l}\text { Environmental } \\
\text { samples analysed } \\
\text { for } 8 \text { PAHs }\end{array}$ \\
\hline
\end{tabular}




\section{$\underline{\text { Association between prenatal } \mathrm{PM}_{2.5} \text { exposure and cognitive and neurobehavioral }}$} development

Chiu et al (2016) examined high $\mathrm{PM}_{2.5}$ exposure during: early, mid and late pregnancy with measures of: full-scale IQ score, inattentiveness and adverse memory performance. Boys highly exposed during late pregnancy exhibit lower $I Q$, and inattentiveness when exposure was from mid to late pregnancy. Girls highly exposed during early to mid-pregnancy exhibited adverse memory performance. No effect was reported for the remaining domains. Contradictory to this Lertxundi et al (2019) reported impaired memory in boys. Lertxundi et al (2015)s finding of impaired motor development was not supported by Lertxundi et al (2019)s report. No effect was found on visual-motor functioning (Blazkova et al. 2020).

No association was observed in cognitive ability (Kerin et al. 2017), general cognitive index (Lertxundi et al. 2019) and mental scale (Lertxundi et al. 2015) nor, non-verbal intelligence (Blazkova et al. 2020), adaptive function, autism spectrum disorder (Kerin et al. 2017) and verbal, perceptive manipulative and numeric development (Lertxundi et al. 2019). Studies reporting prenatal $\mathrm{PM}_{2.5}$ exposure can be found in table 3 . 
Table 3: Studies with measured prenatal $\mathrm{PM}_{2.5}$ exposure on cognitive and neurobehavioral development.

\begin{tabular}{|c|c|c|c|c|c|c|c|}
\hline Citation & $\begin{array}{l}\text { Sample } \\
\text { Size }\end{array}$ & Sample Characteristics & $\begin{array}{l}\text { Male: } \\
\text { Female }\end{array}$ & $\begin{array}{l}\text { Mean } \\
\text { age (SD) }\end{array}$ & $\begin{array}{l}\text { Age } \\
\text { Range } \\
\end{array}$ & $\begin{array}{l}\text { Comorbi } \\
\text { dities }\end{array}$ & $\begin{array}{l}\text { Air Pollution Data } \\
\text { Acquisition Method }\end{array}$ \\
\hline $\begin{array}{l}\text { Blazkova } \\
\text { et al. } \\
2020\end{array}$ & 169 & $\begin{array}{l}\text { Children } 5 \text { years of age, born in the summer } \\
2013 \text { to winter 2014, non-smoking mothers, } \\
\text { resident in Karvina and Ceske Budejovice, } \\
\text { Czech Republic }\end{array}$ & $78: 90$ & $5.5(0.5)$ & $\begin{array}{l}5 \text { years - } \\
5 \text { years } \\
12 \\
\text { months }\end{array}$ & $\begin{array}{l}\text { Viral } \\
\text { Diseases } \\
\text { Otitis } \\
\text { Bronchitis } \\
\text { GIS } \\
\text { HCD }\end{array}$ & $\begin{array}{l}\text { Analysis of } 11 \mathrm{OH}-\mathrm{PAHs} \\
\text { in Urine }\end{array}$ \\
\hline $\begin{array}{l}\text { Kerin et } \\
\text { al. } 2017\end{array}$ & 325 & $\begin{array}{l}\text { Children 2-5 years, resident in catchment area of } \\
20 \text { counties in northern California, the central } \\
\text { valley and parts of Los Angeles metropolitan } \\
\text { area, US, Complete history of Environmental air } \\
\text { exposure, lived with at least } 1 \text { biological parent } \\
\text { who speaks English or Spanish. }\end{array}$ & $281: 44$ & $\begin{array}{l}\text { (not } \\
\text { reported) }\end{array}$ & $\begin{array}{l}2 \text { years - } \\
5 \text { years } \\
12 \\
\text { months }\end{array}$ & $\mathrm{N} / \mathrm{A}$ & $\begin{array}{l}\text { Residential addresses } \\
\text { inputted into Tele Atlas } \\
\text { database and software }\end{array}$ \\
\hline $\begin{array}{l}\text { Lertxundi } \\
\text { et al. } \\
2019\end{array}$ & 560 & $\begin{array}{l}\text { Children } 4 \text { years Male, mothers } \geq 16 \text { years, } \\
\text { resident in Valencia, Sabadell and Gipuzkoa in } \\
\text { Spain }\end{array}$ & $560: 00$ & $4.8(4.9)$ & $\begin{array}{l}4 \text { years - } \\
4 \text { years } \\
12 \\
\text { months }\end{array}$ & $\mathrm{N} / \mathrm{A}$ & $\begin{array}{l}\text { Land use regression } \\
\text { models }\end{array}$ \\
\hline $\begin{array}{l}\text { Lertxundi } \\
\text { et al. } \\
2015\end{array}$ & 438 & $\begin{array}{l}\text { Children aged approx. } 15 \text { months age, mothers } \\
\geq 16 \text { years, singleton pregnancies }\end{array}$ & $198: 240$ & $1.25(0.25)$ & $\begin{array}{l}1 \text { year } 1 \\
\text { month- } 1 \\
\text { year } 6 \\
\text { months }\end{array}$ & $\mathrm{N} / \mathrm{A}$ & $\begin{array}{l}\text { Environmental samples } \\
\text { from Digital DHA-80 high- } \\
\text { volume aerosol samplers }\end{array}$ \\
\hline $\begin{array}{l}\text { Chiu et } \\
\text { al. } 2016\end{array}$ & 119 & $\begin{array}{l}\text { Mothers } \geq 18 \text { years, at } 28.4 \pm 7.9 \text { weeks } \\
\text { gestation between August } 2002 \text { and January } \\
2000 \text { in Boston. }\end{array}$ & $00: 119$ & $6.5(0.98)$ & $\begin{array}{l}6 \text { years - } \\
7 \text { years } 3 \\
\text { months }\end{array}$ & $\mathrm{N} / \mathrm{A}$ & $\begin{array}{l}\text { Use of a hybrid satellite } \\
\text { based spatio-temporal } \\
\text { prediction model and } \\
\text { residential address } \\
\text { during pregnancy }\end{array}$ \\
\hline
\end{tabular}




\section{Childhood}

Association between childhood PAH exposure and general cognition and neurobehavioral development.

Children exposed to high levels of PAH postnatally exhibited lower cognitive ability (Suter et al. 2017). Both Suter et al (2017) and Alemany et al (2018) reported increased inattentiveness, which was contradicted by Mortamais et al (2017). Alemany et al (2018) found an association with impaired working numeric memory, however no effect was reported on working verbal memory. Additionally, Suter et al (2017) reported an effect of delayed impaired memory, but not with short term memory.

The association with ADHD diagnosis, reported by Abid et al (2014) was not supported by 2 studies concerning symptom scores of ADHD (Mortamais et al. 2017; Alemany et al. 2018). No effect to learning performance (Suter et al. 2017), nor learning disability (Abid et al. 2014) was reported. Further to this, no association was found with visual spatial skills, non-verbal test performance, executive function, motor performance (Suter et al. 2017), or behavioural problems (Alemany et al. 2018). Studies reporting childhood PAH exposure can be found in table 4.

Association between childhood $\mathrm{PM}_{2.5}$ exposure and general cognition and neurobehavioral development

Children exposed to high levels of $\mathrm{PM}_{2.5}$ postnatally displayed impaired selective and sustained attention (Saenen et al. 2016). This was not supported by Rivas et al (2019) nor Cserbik et al (2020) reporting no effect on inattentiveness or attention and executive function respectively.

Saenen et al (2016) report of impaired visual information processing speed, was again contradicted by Cserbik et al (2020) reporting no association with processing speed. No association was found with working memory (Rivas et al. 2019; Cserbik et al. 2020), episodic memory, language (Cserbik et al. 2020), cognitive ability, adaptive function or autism spectrum disorder (Kerin et al. 2017). Studies reporting postnatal $\mathrm{PM}_{2.5}$ exposure on general cognition and neurobehavior can be found in table 5 .

Association between childhood $\mathrm{PM}_{2.5}$ exposure and neurodegeneration 
Children highly exposed to $\mathrm{PM}_{2.5}$ postnatally exhibited lower amyloid beta protein fragment 1-42 (A $\left.\beta_{1-42}\right)$. and brain-derived neurotrophic factor (BDNF) (Calderón-Garcidueñas et al. 2018; Calderón-Garcidueñas et al. 2016) and higher interferon (IFN) $\gamma$ concentrations in cerebrospinal fluid (CSF) (Calderón-Garcidueñas et al. 2018). No effect was found with regard to concentrations of biomarkers: non-phosphorylated tau (non-p-tau), vitamin D, tau phosphorylated at threonine 181 (Calderón-Garcidueñas et al. 2018), cellular prion protein, total tau, interleukin (IL) $\beta$, leptin (Calderón-Garcidueñas et al. 2016; Calderón-Garcidueñas et al. 2018), total alpha- synuclein ( $\alpha$-synuclein), oligodendrocyte $\alpha$-synuclein, hyperphosphorylated tau, tumour necrosis factor alpha, IL 2, IL 6, IL 10 or monocyte chemoattractant protein-1 (MCP-1) (Calderón-Garcidueñas et al. 2016). Of 33 antibodies to neural and tight junction proteins actin immunoglobulin $G(\lg G)$, occludin/ zonulin (OZ) immunoglobulin A (IgA), OZ IgG, myelin oligodendrocyte glycoprotein (MOG) IgG, MOG immunoglobulin $M(\operatorname{lgM})$, myelin basic protein (MBP) $\lg A$, MBP $\lg G$, astrocytic protein (S100) IgG, S-100 IgM and cerebellar antigen (CEREB) IgG in serum and MBP antibodies in CSF were higher in children exposed to high levels of PAH compared to controls (CalderónGarcidueñas et al. 2015). Studies reporting postnatal $\mathrm{PM}_{2.5}$ exposure on neurodegeneration can be found in table 6 . 
Table 5: Studies with measured childhood $\mathrm{PM}_{2.5}$ exposure on general cognition and neurobehavioral development.

\begin{tabular}{|c|c|c|c|c|c|c|c|}
\hline Citation & $\begin{array}{l}\text { Sample } \\
\text { Size }\end{array}$ & Sample Characteristics & $\begin{array}{l}\text { Male: } \\
\text { Female }\end{array}$ & $\begin{array}{l}\text { Mean } \\
\text { age (SD) }\end{array}$ & Age Range & $\begin{array}{l}\text { Comorbi } \\
\text { dities }\end{array}$ & $\begin{array}{l}\text { Air Pollution Data } \\
\text { Acquisition Method }\end{array}$ \\
\hline $\begin{array}{l}\text { Cserbik et } \\
\text { al. } 2020\end{array}$ & 10,343 & $\begin{array}{l}\text { Children aged } 9 \text { to } 10 \text { years, resident in one } \\
\text { of } 21 \text { study sites in the US }\end{array}$ & $\begin{array}{l}5410: \\
4933\end{array}$ & $9.93(0.64)$ & $\begin{array}{l}9 \text { years }-10 \\
\text { years and } \\
12 \text { months }\end{array}$ & $\mathrm{N} / \mathrm{A}$ & $\begin{array}{l}\text { Ensemble-based model } \\
\text { approach combining aerosol } \\
\text { optical depth models, land- } \\
\text { use regression, and chemical } \\
\text { transport models }\end{array}$ \\
\hline $\begin{array}{l}\text { Kerin et } \\
\text { al. } 2017\end{array}$ & 325 & $\begin{array}{l}\text { Children } 2-5 \text { years, resident in catchment } \\
\text { area of } 20 \text { counties in northern California, } \\
\text { the central valley and parts of Los Angeles } \\
\text { metropolitan area, US, Complete history of } \\
\text { Environmental air exposure, lived with at } \\
\text { least } 1 \text { biological parent who speaks English } \\
\text { or Spanish }\end{array}$ & $281: 44$ & $\begin{array}{l}\text { (not } \\
\text { reported) }\end{array}$ & $\begin{array}{l}2 \text { years }-5 \\
\text { years } 12 \\
\text { months }\end{array}$ & $\mathrm{N} / \mathrm{A}$ & $\begin{array}{l}\text { Residential addresses } \\
\text { inputted into Tele Atlas } \\
\text { database and software }\end{array}$ \\
\hline $\begin{array}{l}\text { Rivas et } \\
\text { al. } 2019\end{array}$ & 2,221 & $\begin{array}{l}\text { Children 7-10 years old, attending one of } 39 \\
\text { schools in Barcelona, Catalonia, Spain, } \\
\text { without special needs }\end{array}$ & $\begin{array}{l}1133: \\
1088\end{array}$ & $8.5(0.9)$ & $\begin{array}{l}7 \text { years }-10 \\
\text { years } 12 \\
\text { months }\end{array}$ & $\mathrm{N} / \mathrm{A}$ & Land use regression models \\
\hline $\begin{array}{l}\text { Saenen } \\
\text { et al. } \\
2016\end{array}$ & 310 & $\begin{array}{l}\text { Children in grades } 3-6 \text { in three primary } \\
\text { schools, Flanders, Belgium. }\end{array}$ & $\begin{array}{l}158: \\
152\end{array}$ & $10.2(1.3)$ & $\mathrm{N} / \mathrm{A}$ & $\mathrm{N} / \mathrm{A}$ & $\begin{array}{l}\text { Chronic exposure: spatial } \\
\text { temporal interpolation method } \\
\text { to model the daily residential } \\
\text { exposure. } \\
\text { Recent exposure (at schools) } \\
\text { portable devices }\end{array}$ \\
\hline
\end{tabular}


Table 6: Studies with measured childhood $\mathrm{PM}_{2.5}$ exposure on neurodegeneration.

\begin{tabular}{|c|c|c|c|c|c|c|c|}
\hline Citation & $\begin{array}{l}\text { Sample } \\
\text { Size }\end{array}$ & Sample Characteristics & $\begin{array}{l}\text { Male: } \\
\text { Female }\end{array}$ & $\begin{array}{l}\text { Mean } \\
\text { age (SD) }\end{array}$ & $\begin{array}{l}\text { Age } \\
\text { Range }\end{array}$ & $\begin{array}{l}\text { Comorbi } \\
\text { dities }\end{array}$ & $\begin{array}{l}\text { Air Pollution Data } \\
\text { Acquisition Method }\end{array}$ \\
\hline $\begin{array}{l}\text { Calderón- } \\
\text { Garcidueña } \\
\text { s et al. } \\
2018\end{array}$ & 1) 426 & $\begin{array}{l}\text { Children admitted to Mexico City hospital, resident } \\
\text { in Mexico City Metropolitan area (MCMA) and } \\
\text { other small cities in Mexico }\end{array}$ & $\begin{array}{l}\text { 1) } 256: \\
\text { 161 } \\
\text { 2) } 44: 33\end{array}$ & $\begin{array}{l}\text { 1) } 13.36 \\
(8.82) \\
\text { 2) } 11.54 \\
(5.1)\end{array}$ & $\begin{array}{l}\text { (not } \\
\text { reporte } \\
\text { d) }\end{array}$ & $\begin{array}{l}\text { lymphobl } \\
\text { astic } \\
\text { leukaemi } \\
\text { a }\end{array}$ & $\begin{array}{l}\text { Environmental air } \\
\text { sampling, for regulating } \\
\text { levels above the USEPA } \\
\text { standards }\end{array}$ \\
\hline $\begin{array}{l}\text { Calderón- } \\
\text { Garcidueña } \\
\text { s et al. } \\
2016\end{array}$ & 1) 73 & $\begin{array}{l}\text { Children admitted to Mexico City hospital, resident } \\
\text { in Mexico City Metropolitan area (MCMA) and } \\
\text { other small cities in Mexico }\end{array}$ & 1) $42: 31$ & $\begin{array}{l}\text { 1) } 11.7 \\
(5.14) \\
\text { 2) } 17.49 \\
(15.98)\end{array}$ & $\begin{array}{l}\text { (not } \\
\text { reporte } \\
\text { d) }\end{array}$ & $\begin{array}{l}\text { lymphobl } \\
\text { astic } \\
\text { leukaemi } \\
\text { a }\end{array}$ & $\begin{array}{l}\text { Environmental air } \\
\text { sampling }\end{array}$ \\
\hline $\begin{array}{l}\text { Calderón- } \\
\text { Garcidueña } \\
\text { s et al. } \\
2015\end{array}$ & 111 & $\begin{array}{l}\text { Children within } 5 \text { miles of Mexico City Metropolitan } \\
\text { Area(MCMA) or small control cities in Mexico } \\
\text { (Zacatlán and Huachinango, Puebla; Zitaácuaro, } \\
\text { Michoacaán; Puerto Escondido, Oaxaca; Chalma, } \\
\text { Veracruz; Tlaxcala, Tlaxcala), No ETS exposure, } \\
\text { lived within } 5 \text { miles of an air monitoring station }\end{array}$ & $54: 57$ & $\begin{array}{l}13.37 \\
(4.2)\end{array}$ & $\begin{array}{l}\text { (not } \\
\text { reporte } \\
\text { d) }\end{array}$ & $\mathrm{N} / \mathrm{A}$ & $\begin{array}{l}\text { Environmental air } \\
\text { sampling }\end{array}$ \\
\hline
\end{tabular}




\section{Adult}

\section{$\underline{\text { Association between adult PAH exposure and general cognition }}$}

Adults highly exposed to PAH exhibited impaired auditory memory in 2 studies (Du et al. 2020; Niu et al. 2009), and individual accounts of memory disturbances (Ha et al. 2012) and impaired verbal learning and memory (Cho et al. 2020). However, there was no effect on working memory and executive function, visuospatial memory/attention \& planning (Cho et al. 2020) nor a further 2 concurring accounts of no effect on visual perception memory (Du et al. 2020; Niu et al. 2009).

One account of impaired cognitive disturbances (Ha et al. 2012), was contradicted by 2 reports of no association with cognitive dysfunction (Cho et al. 2020; Best et al. 2016). Both Niu et al (2009) and Du et al (2020) reported no association with mood state, attention/response speed, manual dexterity or perceptual motor speed. Additional individual accounts of no effect in approximate number system functioning (Niu et al. 2009), confrontational word retrieval, verbal fluency, delayed reaction time between congruent and incongruent stimuli, visual attention and task switching (Cho et al. 2020) were also reported. All studies involving adult PAH exposure and general cognition can be found in table 7 .

\section{Childhood and Adult}

\section{$\underline{\text { Association between childhood and adult } \mathrm{PM}_{2.5} \text { exposure and neurodegeneration }}$}

In a cohort of mixed exposure to $\mathrm{PM}_{2.5}$, the presence of neurodegenerative biomarkers phosphorylated tau ( $\mathrm{p}$-tau), $\alpha$-synuclein and transactive response DNA binding protein 43 (TDP-43) was confirmed in brainstems (Calderón-Garcidueñas et al. 2020). Faster increase in concentrations with regard to age of non-p-tau in CSF was also associated with increased exposure (Calderón-Garcidueñas et al. 2018). However, no association was found with regard to concentration of total and oligomer a-synuclein in CSF (Calderón-Garcidueñas et al. 2016). Studies reporting on cohorts inclusive of participants exposed to $\mathrm{PM}_{2.5}$ only during childhood and some participants through to adulthood can be found in table 8 .

\section{$\underline{\text { Risk of bias within studies }}$}

All studies included were of a high quality with reproducible accounts of the method employed to assess relevant outcomes, and the inclusion/ exclusion criteria used to select 
the study population explained in sufficient detail. Where applicable all studies provided explanations for participant withdrawal, which were unrelated to both the exposure and the outcome being measured and reported intermediate or unexpected results. $54 \%$ of studies involved the use of a comparison with a low exposure or control population either by dichotomising exposure data or using a demographically matched control population. The remaining $46 \%$ of studies assessed $\mathrm{PAH}$ exposure as a continuous variable. All studies correctly identified confounding variables and the method and analysis was adjusted accordingly. There was however a considerable risk of information bias amongst studies, with only $16 \%$ of studies reporting the outcome assessor to be blinded and unaware of the exposure status of the study participant. $73 \%$ provided no indication as to whether they were or not, and in $11 \%$ the outcome assessors were confirmed unblinded. 
Table 7: Studies with measured adult PAH exposure on general cognition.

\begin{tabular}{|c|c|c|c|c|c|c|c|}
\hline Citation & $\begin{array}{l}\text { Sample } \\
\text { Size }\end{array}$ & Sample Characteristics & $\begin{array}{l}\text { Male: } \\
\text { Female }\end{array}$ & $\begin{array}{l}\text { Mean } \\
\text { age } \\
\text { (SD) }\end{array}$ & $\begin{array}{l}\text { Age } \\
\text { Range }\end{array}$ & Comorbidities & $\begin{array}{l}\text { Air Pollution Data } \\
\text { Acquisition Method }\end{array}$ \\
\hline $\begin{array}{l}\text { Du et al. } \\
2020\end{array}$ & 697 & $\begin{array}{l}\text { Employed at a coking plant in Shanxi } \\
\text { province, China for minimum of } 1 \text { year }\end{array}$ & $470: 227$ & $\begin{array}{l}39.73 \\
(9.74)\end{array}$ & $\begin{array}{l}24-64 \\
\text { years } 12 \\
\text { months }\end{array}$ & $\mathrm{N} / \mathrm{A}$ & $\begin{array}{l}\text { The sum of the } \\
\text { concentrations of eleven } \\
\text { urinary PAHs } \\
\text { metabolites } \Sigma \text {-OHPAHs }\end{array}$ \\
\hline $\begin{array}{l}\text { Cho et al. } \\
2020\end{array}$ & 949 & $\begin{array}{l}\geq 50 \text { year-old individuals, no known } \\
\text { neurological diseases, resident in Seoul, } \\
\text { Incheon, Wonju and Pyeongchang, } \\
\text { Republic of Korea. }\end{array}$ & $421: 528$ & $\begin{array}{l}67.24 \\
(6.39)\end{array}$ & $\geq 50$ years & $\begin{array}{l}\text { Hypertension } \\
\text { Diabetes } \\
\text { Dyslipidaemia } \\
\text { Angina Myocardial } \\
\text { infarction }\end{array}$ & $\begin{array}{l}\text { Concentrations of } \\
\text { urinary PAHs } \\
\text { metabolites including: 1- } \\
\text { hydroxypyrene }\end{array}$ \\
\hline $\begin{array}{l}\text { Ha et al. } \\
2012\end{array}$ & 565 & $\begin{array}{l}\text { Volunteers in the Hebei Spirit oil spill, } \\
2007 \text {, near the shore of Taean, Korea. }\end{array}$ & $275: 288$ & $\mathrm{~N} / \mathrm{A}$ & $\mathrm{N} / \mathrm{A}$ & Asthma & $\begin{array}{l}\text { 1-hydroxypyrene and 2- } \\
\text { naphthol metabolites in } \\
\text { urine }\end{array}$ \\
\hline $\begin{array}{l}\text { Niu et al. } \\
2009\end{array}$ & 176 & $\begin{array}{l}\text { Male 23-48-year-old coke oven workers } \\
\text { Taiyuan, China, employed for a minimum of } \\
\text { 1-year, middle school educated. }\end{array}$ & 176:00 & $\begin{array}{l}37.86 \\
(6.61)\end{array}$ & $\begin{array}{l}23 \text { years } \\
\text { to } 48 \\
\text { years } 12 \\
\text { months }\end{array}$ & $\mathrm{N} / \mathrm{A}$ & $\begin{array}{l}\text { Concentration of urinary } \\
\text { PAH metabolite 1- } \\
\text { hydroxypyrene (1-OHP) }\end{array}$ \\
\hline $\begin{array}{l}\text { Best et al. } \\
2016\end{array}$ & 454 & $\begin{array}{l}\geq 60 \text {-year-old individuals without known } \\
\text { neurological diseases, resident in } 15 \\
\text { randomly selected states in the US }\end{array}$ & $221: 233$ & $\begin{array}{l}70.1 \\
(0.02)\end{array}$ & $\begin{array}{l}\geq 60 \\
\text { years }\end{array}$ & $\begin{array}{l}\text { Hypertension } \\
\text { Thyroid Disease } \\
\text { Stroke } \\
\text { Kidney Disease } \\
\text { Liver Disease }\end{array}$ & $\begin{array}{l}\text { The sum of the } \\
\text { concentrations of eight } \\
\text { urinary PAHs } \\
\text { metabolites ( } \Sigma- \\
\text { OHPAHs) }\end{array}$ \\
\hline
\end{tabular}


Table 8: Studies with measured cohorts inclusive of childhood and adult PM2.5 exposure on Neurodegeneration

\begin{tabular}{|c|c|c|c|c|c|c|c|}
\hline Citation & $\begin{array}{l}\text { Sample } \\
\text { Size }\end{array}$ & Sample Characteristics & $\begin{array}{l}\text { Male: } \\
\text { Female }\end{array}$ & $\begin{array}{l}\text { Mean } \\
\text { age (SD) }\end{array}$ & Age Range & Comorbidities & $\begin{array}{l}\text { Air Pollution Data } \\
\text { Acquisition Method }\end{array}$ \\
\hline $\begin{array}{l}\text { Calderón- } \\
\text { Garcidueñas } \\
\text { et al. } 2020\end{array}$ & 186 & $\begin{array}{l}\text { Metropolitan Mexico City residents, } \\
\text { acute cause of death not involving the } \\
\text { brain, autopsies were performed } 3.7 \pm \\
1.7 \mathrm{~h} \text { after death, autopsy material } \\
\text { examined between } 2004 \text { and } 2008\end{array}$ & $162: 186$ & $\begin{array}{l}27.29 \\
(11.8)\end{array}$ & $\begin{array}{l}11 \text { months- } \\
40 \text { years } 12 \\
\text { months }\end{array}$ & $\mathrm{N} / \mathrm{A}$ & $\begin{array}{l}\text { Ministry of } \\
\text { Environment of } \\
\text { Mexico City monitoring } \\
\text { stations }\end{array}$ \\
\hline $\begin{array}{l}\text { Calderón- } \\
\text { Garcidueñas } \\
\text { et al. } 2018\end{array}$ & $\begin{array}{l}\text { 1) } 426 \\
\text { 2) } 81\end{array}$ & $\begin{array}{l}\text { Children admitted to Mexico City } \\
\text { hospital, resident in Mexico City } \\
\text { Metropolitan area (MCMA) and other } \\
\text { small cities in Mexico }\end{array}$ & $\begin{array}{l}\text { 1) } 256: \\
\text { 161 } \\
\text { 2) } 44: 33\end{array}$ & $\begin{array}{l}\text { 1) } 13.36 \\
(8.82) \\
\text { 2) } 11.54 \\
(5.1)\end{array}$ & $\mathrm{N} / \mathrm{A}$ & $\begin{array}{l}\text { lymphoblastic } \\
\text { leukaemia }\end{array}$ & $\begin{array}{l}\text { Environmental air } \\
\text { sampling, for } \\
\text { regulating levels } \\
\text { above the USEPA } \\
\text { standards }\end{array}$ \\
\hline $\begin{array}{l}\text { Calderón- } \\
\text { Garcidueñas } \\
\text { et al. } 2016\end{array}$ & $\begin{array}{l}\text { 1) } 73 \\
\text { 2) } 126\end{array}$ & $\begin{array}{l}\text { Children admitted to Mexico City } \\
\text { hospital, resident in Mexico City } \\
\text { Metropolitan area (MCMA) and other } \\
\text { small cities in Mexico }\end{array}$ & $\begin{array}{l}\text { 1) } 42: 31 \\
\text { 2) } 59: 70\end{array}$ & $\begin{array}{l}\text { 1) } 11.7 \\
(5.14) \\
\text { 2) } 17.49 \\
(15.98)\end{array}$ & $\mathrm{N} / \mathrm{A}$ & $\begin{array}{l}\text { lymphoblastic } \\
\text { leukaemia }\end{array}$ & $\begin{array}{l}\text { Environmental air } \\
\text { sampling }\end{array}$ \\
\hline
\end{tabular}




\section{Discussion}

This review found sufficient evidence that prenatal PAH exposure negatively impacts cognitive function with specific regard to child intelligence, mental development, verbal IQ, memory impairment, average overall development, child attentiveness, behavioural development and externalizing, internalizing, anxious and depressed behavioural problems. Evidence concerning exposure during childhood and as an adult with cognitive function was insufficient to conduct a meta-analysis, due to a reduced number of studies, low consistency and high heterogeneity in results. However, associations can be observed such as exposure during childhood with lowered cognitive ability, and impaired child attentiveness, and exposure as an adult manifesting in memory disturbances with specific regard to auditory memory and verbal learning and memory.

Studies concerning PAH exposure during childhood, and as an adult were scarce, but found an increased risk of neurodegeneration via the presence of neurodegenerative biomarkers and increased concentrations of cryptic "self" antigens in serum and CSF, indicative of the neuroinflammatory pathology which precedes Alzheimer's disease (AD) and Parkinson's disease (PD).

This review disentangled the neurological impact of PAHs from other polluting compounds and can be used as evidence for policy surrounding the monitoring of PAHs specifically. In addition, it raises awareness of the potentially confounding effect, that different ambient PAH concentrations, in metropolitan and rural settings, can have on research assessing outcomes concerned with cognitive function and neurodegeneration in studies.

A previous review on the impact of PM2.5 in disease incidence did not stratify patients by age and neither considered differences between urban and rural areas, rather stratifying studies by the pollution level in which the country was considered (i.e., "lightly polluted" vs. "heavily polluted") (Fu et al., 2019). Other reviews have highlighted general adverse health conditions such as chronic asthma, increased incidence of premature death and hospital admissions (Abdel-Shafy \& Mansour, 2016), and kidney and liver damage (Kim et al. 2013). Some focussed specifically on the carcinogenic properties and resulting incidence of lung (Armstrong et al. 2004), urinary tract (Bosetti et al. 2007), skin and gastrointestinal tract cancers (Kim et al. 2013). Those that focussed on the neurological impact of air pollution, concerned a diverse mixture of compounds. Peters et al (2019) specifically focus on noncommunicable diseases and the roles of Nitrogen dioxide (NO2), Nitrogen oxide species (NOx), Carbon monoxide (CO) and PM2.5. Schikowski \& Altug, (2020) raise awareness concerning ambient pollutions adverse effect on cognitive decline and impairment, 
concurring with findings from Calderón-Garcidueñas et al, (2016), where the emphasis was on ozone, PM2.5 and PM10. Kilian \& Kitazawa, (2018) report NO2, NOx, black carbon and PMs role as potential risk factors for AD, PD and multiple sclerosis. Despite the outcomes assessed being orientated toward neurological health, the exposures measured either include multiple pollutants, or compounds NO2, NOx, CO, PM or black carbon, around which extensive research already exists and has culminated in tight air quality restrictions and monitoring, which is closely adhered to by governing bodies. This review raises awareness as to the neurological impact, PAH has independent of other pollutants, the importance of which is paramount with the current health effects of PAHs in the UK Air quality strategy detailed as "possibly" or "probably" carcinogenic, detracting from the seriousness of their impact on neurological human health (Department for Environment, Food and Rural Affairs, 2011). This review proceeds to categorise outcomes into subgroups depending on the time of exposure to provide further insight into the demographic most vulnerable and to differentiate between the areas of cognitive function and neurodegeneration most impacted, elucidating the potential mechanisms of neurotoxicity. Observation that the most profound effect of PAH exposure culminates from the prenatal period is in keeping with prior research, showing the fetal brain to be more vulnerable to environmental toxic insult than the adult. The increased permeability of the not yet fully formed blood-brain barrier combined with the rapid brain growth during the second trimester, means the period of most intense construction and brain architecture is also the time the brain is most vulnerable to passage of toxins and neurotoxicity (Lanphear BP, 2015).

\section{Limitations}

Studies included in the analysis were limited to those written in the English language. Publication bias, and selective reporting within studies cannot be discarded, nor can indexing issues, in which the search terms failed to identify relevant studies. The study populations included only originated from 9 countries, of which the UK was not one. Findings are therefore limited to the environments and seasonal variations in climate found in these countries and no specific recommendations for UK can be made. Studies included also involved the use of different subgroup samples from the same large cohort, due to the necessity and availability of a limited number of longitudinal study databases. Sampling bias cannot therefore be disregarded.

To adjust for heterogeneity studies were stratified depending on the time point of exposure and outcome assessed, however this did not account for heterogeneity between evaluators and instruments used. In addition to this the use of 5 different measures to quantify levels of $\mathrm{PAH}$ exposure as well as the inclusion of quantification of $\mathrm{PM}_{2.5}$ as a measure, resulted in 
heterogeneity in exposure measurement instruments, and the inclusion of potential contaminating compounds within $\mathrm{PM}_{2.5}$ which would confound results. Finally, there was insufficient data to calculate $95 \% \mathrm{Cl}$ for 1 study (Perera et al. 2015) and the request for numeric data from another study, received no response, hence the report of an effect on memory and cognitive disturbances was inferred from a figure with no confirmation from the raw data (Ha et al. 2012).

\section{Future Research}

The role of gender in the neurotoxic effects of PAH exposure requires further investigation. Sex stratification of data concerning memory impairment in prenatally exposed populations was contradictory. Further accounts of memory impairment following both childhood and adult exposure should be dichotomized according to gender to examine sensitivity between sexes. Prenatal PAH exposure effect on motor development was an area of controversy. Additional research is required in this domain to eliminate ambiguity, as well as further analysis following childhood and adult exposure. Individual reports of a lack of association with motor performance and perceptual motor speed respectively, were inadequate to clarify such controversy, or draw any conclusions.

In addition to this a more thorough examination of the timescale of PAH exposure is needed, utilising a smaller scale to determine critical windows. Stratification by pregnancy term elucidated to differential full-scale IQ, inattentiveness and memory performance results. No effect on concentration of non-p-tau in CSF was reported following childhood exposure, however when looked at in a mixed cohort of childhood and adult exposure, in relation to age progression an association was reported, indicative of a critical window of exposure. Further to this gene-environment interactions need further analysis, PAHs effect on the brain of genetically susceptible populations, such as carriers of the APOE4 allele.

Repeated future analysis on longitudinal cohorts is required to examine the impact of sustained high PAH exposure or subsequent markedly reduced exposure, and the effects such fluctuations can have on cognitive function and neurodegeneration and whether some adverse effects from prenatal or early life exposure are recoverable.

Future research should identify and analyse the individual contributions, and specific synergistic combinations of PAHs on neurological health. This would differentiate and determine the most neurotoxic PAHs, and provide evidence for an update in policy, requiring the monitoring of additional PAHs other than $\mathrm{B}[\mathrm{a}] \mathrm{P}$. Additional research into the threshold at which $\mathrm{PAH}$ is capable of exerting neurotoxic effects would inform policy, with scientific backing on a safe limit with regard to neurological health and update the limit of $0.25 \mathrm{ng} \cdot \mathrm{m}^{-3}$ $\mathrm{B}[\mathrm{a}] \mathrm{P}$ set with regard only, to the carcinogenic properties. Further to this more studies are 
needed concerning populations in the UK, to account for the local environment, climate and seasonal variations, capable of altering PAH's neurotoxic properties.

\section{Acknowledgements}

$\mathrm{JH}$ is funded by the College of Medicine and Veterinary Medicine at The University of Edinburgh and MVH is funded by The Row Fogo Charitable Trust (Grant No. BROD.FID3668413).

\section{$\underline{\text { References }}$}

Abdel-Shafy H. \& Mansour M. S (2016). A review on polycyclic aromatic hydrocarbons. Environmental impact, effect on human health and remediation. Egyptian Journal of Petroleum 25, 107-123.

Abid Z, Roy A, Herbstman JB, Ettinger AS (2014). Urinary Polycyclic Aromatic Hydrocarbon Metabolites and Attention/Deficit Hyperactivity Disorder, Learning Disability, and Special Education in U.S. Children Aged 6 to 15. Journal of Environmental and Public Health 2014, $1-10$.

ACGIH, 2005. Polycyclic aromatic hydrocarbons (PAHs) biologic exposure indices (BEI) Cincinnati, Cincinnati: American Conference of Governmental Industrial Hygienists (ACGIH)

Alemany S, Vilor-Tejedor N, García-Esteban R, Bustamante M, Dadvand P, Esnaola M, Mortamais M, Forns J, van Drooge B, Álvarez-Pedrerol M et al (2018). Traffic-related air pollution, APOE E4 status, and neurodevelopmental outcomes among school children enrolled in the BREATHE Project (Catalonia, Spain). Environmental Health Perspectives 126, 087001-1-11.

Armstrong B, Hutchinson E, Unwin J, Fletcher T (2004) . Lung cancer risk after exposure to polycyclic aromatic hydrocarbons. A review and meta-analysis. Environmental Health Perspectives 112, 970-978.

Bach P B, Kelley M J, Tate R C, McCrory D C (2003). Screening for lung cancer: a review of the current litrature. Chest 123, 1. 
Best EA, Juaez-Colunga E, James K, LeBlanc WG, Serdar B (2016). Biomarkers of exposure to polycyclic aromatic hydrocarbons and cognitive function among elderly in the United States. National Health and Nutrition Examination Survey: 2001-2002 PLOS one 11, $1-18$.

Blazkova B, Pastorkova A, Solansky I, Veleminsky Jr M, Veleminsky M, Urbancova K, Vondraskova V, Hajslova J, Pulkrabova J \& Sram RJ (2020). Effect of polycyclic aromatic hydrocarbons exposure on cognitive development in 5 years old children. Brain Sciences 10, 619-630.

Boffetta P, Jourenkova N, Gustazsson P (1997). Cancer risk from occupational and environmental exposure to polycyclic aromatic hydrocarbons. Cancer Causes Control 8, 3.

Bosetti C, Boffetta, P, La Vecchia, C (2007). Occupational exposures to polycyclic aromatic hydrocarbons, and respiratory and urinary tract cancers: a quantitative review to 2005. Annals Oncology 2007 Mar 18, 431-446.

Calderón-Garcidueñas L, Avila- Ramírez J, Calderón-Garcidueñas A, Gonzáles-Heredia T, Acuña- Ayala H, Chao C, Thompson C, Ruiz-Ramos R, Cortés-González V, MartínezMartínez $L$ et al (2016). Cerebrospinal fluid biomarkers in highly exposed $\mathrm{PM}_{2.5}$ urbanites: The risk of Alzheimer's and Parkinson's Diseases in Young Mexico City Residents. Journal of Alzheimer's Disease 54, 597-613.

Calderón-Garcidueñas L, González- Maciel A, Reynoso-Robles R, Hammond J, Kulesza R, Lachmann I, Torres-Jardón R, Mukherjee PS, Maher BA (2020). Quadruple abnormal protein aggregates in brainstem pathology and exogenous metal-rich magnetic nanoparticles (and engineered Ti-rich nanorods). The substantia nigrae is a very early target in young urbanites and the gastrointestinal tract a key brainstem portal. Environmental Research 191, 1-17.

Calderón-Garcidueñas L, Leray E, Heydarpour P, Torres-Jardón R, Reis J (2016). Air pollution, a rising environmental risk factor for cognition, neuroinflammation and neurodegeneration. The clinical impact on children and beyond. Revue Neurologique 172, 69-80.

Calderón-Garcidueñas L, Mukherjee PS, Waniek K, Holzer M, Chao C, Thompson C, RuizRamos R, Calderón-Garcidueñas A, Franco-Lira M, Reynoso-Robles R et al (2018). Non- 
phosphorylated Tau in cerebrospinal fluid is a marker of Alzheimer's Disease continuum in young urbanites exposed to air pollution. Journal of Alzheimer's Disease 66, 1437-1451.

Calderón-Garcidueñas L, Vojdani A, Blaurock- Busch E, Busch Y, Friedle A, Franco-Lira M, Mukherjee PS, Martínez-Aguirre X, Park SB, Torres-Jardón R, D’Angiulli A (2015). Air pollution and children: Neural and tight junction antibodies and combustion metals, the role of barrier breakdown and brain immunity in neurodegeneration. Journal of Alzheimer's Disease 43, 1037-1058.

Cao X, Li J, Cheng L, Deng Y, Li Y, Yan Z, Duan L, Yang J, Niu Q, Perera F, Nie J, Tang D (2020). The associations between prenatal exposure to polycyclic aromatic hydrocarbon metabolites, umbilical cord blood mitochondrial DNA copy number, and children's neurobehavioral development. Environmental Pollution 265, 1-10.

Chiu YM, Hsu HL, Coull BA, Bellinger DC, Kloog I, Schartz J, Wright RO, Wright RJ (2016). Prenatal particulate air pollution and neurodevelopment in urban children: Examining sensitive windows and sex-specific associations. Environment International 87, 56-65.

Cho J, Sohn J, Noh J, Jang H, Kim W, Cho SK, Seo H, Seo G, Lee SK, Noh Y et al (2020). Association between exposure to polycyclic aromatic hydrocarbons and brain cortical thinning: The environmental pollution-induced neurological effects (EPINEF) study. Science of the Total Environment 737, 1-9.

Cohen A J, Brauer M, Burnett R, Anderson H R, Frostad J, Estep K, Balakrishnan K, Brunekeef B, Dandona L, Dandona R (2017). Estimates and 25-year trends of the global burden of disease attributable to ambient air pollution: an analysis of data from the Global Burden of Diseases Study 2015. The Lancet 389, 1907-1918.

Cserbik D, Chen JC, McConnell R, Berhane K, Sowell ER, Schwartz J, Hackman DA, Kan E, Fan CC, Herting MM (2020). Fine particulate matter exposure during childhood relates to hemispheric- specific differences in brain structure. Environment International 143, 1-11.

Department for Environment, Food and Rural Affairs, 2011. The Air Quality Strategy for England, Scotland, Wales and Northern Ireland, Norwich: HMSO.

Department of the Environment, Food and Rural Affairs , 2020. Air Pollution in the UK 2019, Norwich: HMSO. 
Diggs D L, Harris K L, Rekhadevi P V, Ramesh A (2012). Tumor microsomal metabolism of the food toxicant, benzo(a)pyrene, in ApcMin mouse model of colon cancer. Tumour Biol 33, $1-12$.

Du J, Pan B, Cao X, Li J, Yang J, Nie J (2020). Urinary polycyclic aromatic hydrocarbon metabolites, peripheral blood mitochondrial DNA copy number and neurobehavioral function in coke oven workers. Chemosphere 261, 1-11.

Edwards SC, Jedrychowski W, Butscher M, Camann D, Kieltyka A, Mroz E (2010). Prenatal exposure to airborne polycyclic aromatic hydrocarbons and children's intelligence at 5 years of age in a prospective cohort study in Poland. Environmental Health Perspectives 118, 1-9.

Finardi S, Radice P, Cecinato A, Gariazzo C, Gherardi M, Romagnoli P (2017). Seasonal variation of PAHs concentration and source attribution through diagnostic ratios analysis. Urban Climate 22, 19-34.

Fu P, Guo X, Cheung FMH, Yung KKL (2019). The association between PM2.5 exposure and neurological disorders: A systematic review and meta-analysis. Science of the Total Environment $1655,1240-1248$.

Ha M, Kwon H, Cheong HK, Lim S, Yoo SJ, Kim EJ, Park SG, Lee J, Chung BC (2012). Urinary metabolites before and after clean-up and subjective symptoms in volunteer participants in clean-up of the Hebei Spirit oil spill. Science of the Total Environment 429, 167-173.

Hartz A M S, Bauer B, Block M L, Hong J-S, Miller D S (2008). Diesel exhaust particles induce oxidative stress, proinflammatory signaling, and $\mathrm{P}$-glycoprotein up-regulation at the blood-brain barrier. The FASEB Journal 22(8), 2723-2733.

Hassanvand M S, Naddafi K, Faridi S, Nabizadeh R, Sowlat M H, Momeniha F, Gholampour A, Arhami M, Kashani H, Zare A, Niazi S, Rastkari N, Nazmara S, Ghani M (2015). Characterization of PAHs and metals in indoor/outdoor PM10/PM2.5/PM1 in a retirement home and a school dormitory. Science of The Total Environment 527-528(15), 100-110.

Jedrychowski W, Perera F, Camann D, Spengler J, Butscher M, Mroz E, Majewska R, Flak E, Jacek R, Sowa A (2015). Prenatal exposure to polycyclic aromatic hydrocarbons and cognitive dysfunction in children. Environmental Science Pollution Research 22, 3631- 3639. 
Kerin T, Volk H, Li W, Lurmann F, Eckel S, McConnell R, Hertz-Picciotto (2017). Association between air pollution exposure, cognitive and adaptive function, and ASD severity among children with autism spectrum disorder. Journal of Autism and Developmental Disorders 48, 137-150.

Kim K H, Ara J S, Kabir E, Brown RJ (2013). A review of airborne polycyclic aromatic hydrocarbons (PAHs) and their human health effects. Environment International 60, 71-80.

Kilian, J. \& Kitazawa, M., 2018. The emerging risk of exposure to air pollution on cognitive decline and Alzheimer's disease- Evidence from epidemiological and animal studies. Biomedical Journal, Volume 41, pp. 141-162.

Kuo C-Y, Chien P S, Kuo W-C, Wei C-T, Rau J-Y (2012). Comparison of polycyclic aromatic hydrocarbon emissions on gasoline- and diesel-dominated routes. Environmental Monitoring and Assesment 185, 5749-5761.

Lanphear BP, (2015). The Impact of Toxins on the Developing Brain. Annual Review of Public Health 36, 211-230.

Lertxundi A, Andiarena A, Martínez MD, Ayerdi M, Murcia M, Estarlich M, Guxens M, Sunyer J, Julvez J, Ibarluzea J (2019). Prenatal exposure to $\mathrm{PM}_{2.5}$ and $\mathrm{NO}_{2}$ and sex-dependent infant cognitive and motor development. Environmental Research 174, 114-121.

Lertxundi A, Baccini M, Lertxundi N, Fano E, Aranbarri A, Martínez MD, Ayerdi M, Álvarez J, Loreto SM, Dorronsoro M, Ibarluzea J (2015). Exposure to fine particle matter, nitrogen dioxide and benzene during pregnancy and cognitive and psychomotor developments in children at 15 months of age. Environment International 80, 33-40.

Liberati A, Altman D G, Tetzlaff J, Mulrow C, Gøtzsche P C, Loannidis J P A, Clarke M, Devereaux P J, Kleijnen J, Moher D (2009). The PRISMA statement for reporting systematic reviews and meta-analyses of studies that evaluate healthcare interventions: explaination and elaboration. British Medical Journal 339, 1-14 .

Mortamais M, Pujol J, van Drooge BL, Macià D, Martínez-Vilavella G, Reynes C, Sabatier R, Rivas I, Grimalt J, Forns J, Alvarez-Pedrerol M, Querol X, Sunyer J (2017). Effect of exposure to polycyclic aromatic hydrocarbons on basal ganglia and attention-deficit hyperactivity disorder symptoms in primary school children. Environment International 105, 12-19. 
Nie J, Li J, Cheng L, Deng Y, Li Y, Yan Z, Duan L, Niu Q, Tang D (2019). Prenatal polycyclic aromatic hydrocarbons metabolites, cord blood telomere length, and neonatal neurobehavioral development. Environmental Research 174, 105-113.

Niu Q, Zhang H, Li X, Li M (2010). Benzo[a]pyrene-induced neurobehavioral function and neurotransmitter alterations in coke oven workers. Occupational and Environmental Medicine 67, 444-448.

Olsson A C, Fevotte J, Fletcher T, Cassidy, A, Mannetje A, Zaridze D, SzeszeniaDabrowska N Ridnai P, Lissowska J, Fabianova E, Mates D, Bencko V, Foretova L, Janout $\mathrm{V}$ (2010). Occupational exposure to polycyclic aromatic hydrocarbons and lung cancer risk: a multicenter study in Europe. Occup Environ Med 67(2) 98-103.

Pagliaccio D, Herbstman JB, Perera F, Tang D, Goldsmith J, Peterson BS, Rauh V, Margolis AE (2020). Prenatal exposure to polycyclic aromatic hydrocarbons modifies the effects of early life stress on attention and thought problems in late childhood. The Journal of Child Psychology and Psychiatry 61, 1253-1265.

Perera F, Li T, Zhou Z, Yuan T, Chen Y, Qu L (2008). Benefits of reducing prenatal exposure to coal-burning pollutants to children's neurodevelopment in China. Environmental Health Perspectives 116, 1396-1403.

Perera F, Phillips DH, Wang Y, Roen E, Herbstman J, Rauh V, Wang S, Tang D (2015). Prenatal exposure to polycyclic aromatic hydrocarbons/aromatics, BDNF and child development. Environmental research 142, 602-608.

Perera F, Rauh V, Whyatt RM, Tsai WY, Tang D, Diaz D (2006). Effect of prenatal exposure to airborne polycyclic aromatic hydrocarbons on neurodevelopment in the first 3 years of life among inner-city children. Environmental Health Perspectives 114, 1287-1298.

Perera F, Tang D, Wang S, Vishnevetsky J, Zhang B, Diaz D (2012). Prenatal polycyclic aromatic hydrocarbon (PAH) exposure and child behavior at age 6-7 years. Environmental Health Perspectives 120, 921-930.

Perera F, Wang S, Rauh V, Zhou H, Stigter L, Camann D, Jedrychowski W, Mroz E, Majewska R (2013). Prenatal Exposure to Air Pollution, maternal psychological distress, and child behavior. Pediatrics 132, 1284-1294. 
Perera F, Wang S, Vishnevetsky J, Zhang B, Cole K, Tang D, Rauh V, Philips D (2011). Polycyclic aromatic hydrocarbons-aromatic DNA adducts in cord blood and behaviour scores in New York City children. Environmental Health Perspectives 119, 1176-1181.

Perera F, Wheelock K, Wang Y, Tang D, Margolis AE, Badia G, Cowell W, Miller RL, Rauh V, Wang S, Herbstman JB (2018). Combined effects of prenatal exposure to polycyclic aromatic

hydrocarbons and material hardship on child ADHD behavior problems. Environmental research 160, 505-513.

Peters R, Ee N, Peters J, Booth A, Mudway I (2019). Air Pollution and Dementia: A Systematic Review. Journal of Alzheimer's Disease 70, 145-164.

Polanska K, Hanke W, Sobala W, Trzcinka-Ochocka M, Ligocka D, Brzeznicki S, StrugalaStawik H, Magnus P (2013). Developmental effects of exposures to environmental factors: The Polish mother and child cohort study. BioMed Research International 2013, 1-11.

Rivas I, Basagaña X, Cirach M, López-Vicente M, Suades-González E, Garcia-Esteban R, Álvarez-Pedrerol M, Dadvand P, Sunyer J (2019). Association between Early Life Exposure to Air Pollution and Working Memory and Attention. Environmental Health Perspectives 127, 05700-1-05700-11.

Saenen ND, Provost EB, Viaene MK, Vanpoucke C, Lefebvre W, Vrijens K, Roels HA. Nawrot TS (2016). Recent versus chronic exposure to particulate matter air pollution in association with neurobehavioral performance in a panel study of primary schoolchildren. Environment International 95, 112-119.

Saunders C R, Das S K, Ramesh A, Shockley D C, Mukherjee S (2006). Benzo(a)pyreneinduced acute neurotoxicity in the F-344rat: role of oxidative stress. Journal of Applied Toxicology 26, 427-438.

Schikowski T. \& Altug, H. (2020). The role of air pollution in cognitive impairment and decline. Neurochemistry International 136, 1-4.

Suter MK, Karr CJ, John-Stewart GC, Gómez LA, Moraa H, Nyatika D, Wamalwa D, Paulsen M, Simpson CD, Boivin MJ, Bangirana P, Benki-Nugent S (2018). Implications of combined exposure to household air pollution and HIV on neurocognition in children. Environment Research and Public Health 15, 163-176. 
Tang D, Lee J, Muirhead L, Li TY, Qu L, Yu J, Perera F (2014). Molecular and neurodevelopmental benefits to children of closure of a coal burning power plant in China. PLOS one 9, 1-6.

Tang D, Li TY, Chow JC, Kulkarni SU, Watson JG, Ho SSH, Quan ZY, Perera F (2014). Air pollution effects on fetal and child development: A cohort comparison in China. Environmental Pollution 185, 90-96.

Tang D, Li T, Liu JJ, Zhou Z, Yuan T, Chen Y (2008). Effects of prenatal exposure to coalburning pollutants on children's development in China. Environmental Health Perspectives 116, 674- 683.

The European Parliament and The European Council of the European Union 2015. Directive 2004/107/EC of the European Parliament and of the Council of 15December 2004 relating to arsenic, cadmium, mercury, nickel and polycyclic aromatic hydrocarbons in ambient air, Brussels. The European Parliament and The Council of the European Union.

U.S. Department of health and and human services, 1995. Toxicological profile for polycyclic aromatic hydrocarbons. Agency for Toxic substances and Disease Registry.

Zhang Y, Yang Y, Zhang Q, Cui J, Rahaman A, Huang X, Su Y, Gao R, Wang B, Baloch Z (2019). Effect of Benzo[a]pyrene-DNA adduct in cord blood on the neurodevelopment of 12month-old infants in Qingdao City. Neuropsychiatric Disease and Treatment 15, 3351-3357.

\section{Web References}

QUADAS-2. URL

http://www.bristol.ac.uk/population-health-sciences/projects/quadas/quadas-2/ 